\title{
ANALYSIS OF A MATHEMATICAL MODEL OF THE EFFECT OF INHIBITORS ON THE GROWTH OF TUMORS
}

\author{
SHANGBIN CUI* AND AVNER FRIEDMAN**
}

\begin{abstract}
*Department of Mathematics, Lanzhou University, Lanzhou, Gansu 730000, People's Republic of China and Institute for Mathematics and its Applications, University of Minnesota, Minneapolis, MN 55455.
\end{abstract}

** Department of Mathematics, University of Minnesota, 206 Church St. SE, Minneapolis, MN 55455.

\begin{abstract}
In this paper we study a model of tumor growth in the presence of inhibitors. The tumor is assumed to be spherically symmetric and its boundary is an unknown function $r=R(t)$. Within the tumor the concentration of nutrient and the concentration of inhibitor (drug) satisfy a system of reaction diffusion equations. The important parameters are $\Lambda_{0}$ (which depends on the tumor's parameters when no inhibitors are present), $\gamma$ which depends only on the specific properties of the inhibitor, and $\bar{\beta}$ which is the (normalized) external concentration of the inhibitor. In this paper we give precise conditions under which there exist one dormant tumor, two dormant tumors, or none. We then prove that in the first case, the dormant tumor is globally asymptotically stable, and in the second case, if the radii of the dormant tumors are denoted by $R_{s}^{-}, R_{s}^{+}$with $R_{s}^{-}<R_{s}^{+}$, then the smaller one is asymptotically stable, so that $\lim _{t \rightarrow \infty} R(t)=R_{s}^{-}$, provided the initial radius $R_{0}$ is smaller than $R_{s}^{+}$; if however $R_{0}>R_{s}^{+}$then the initial tumor in general grows unboundedly in time. The above analysis suggests an effective strategy for treatment of tumors.
\end{abstract}

Key words. tumors, inhibitors, parabolic equations, free boundary problems.

1. The model. In this paper we study a model recently proposed by Byrne and Chaplain [1] for the growth of tumor in the presence of inhibitors. The tumor consists of life cells (nonnecrotic tumor) and receives blood supply through a developed network of capillary vessels (vascularized tumor). The blood supply provides the tumor with nutrients as well as inhibitors. Inhibitors may also diffuse into the tumor from neighboring tissues. The inhibitors may develop from the immune system of healthy cells, as well as from drugs administered for therapy.

The paper develops mathematical techniques for rigorous analysis of transient and stationary solutions to such models. In the particular model under study they allow us to confirm, but also significantly extend, the results obtained in [1] through numerical studies.

As in other models developed over the last 30 years (see, e.g., [2-6] and the references cited there), Byrne and Chaplain represent the tumor's evolution in the form of a free-boundary problem whereby its growth is determined by the levels of diffusing nutrient and inhibitor concentrations. In contrast with previous ones, however, their model departs from in vitro growth scenarios by taking into account the possible blood-tissue nutrient transfer that occurs in vivo through angiogenesis as described and modelled in [7], [8, chap. 5]. (Angiogenesis is a process by which tumors induce blood vessels to sprout capillary tips which migrate toward, and penetrate into, the tumor thus providing it with circulating blood supply.) Further, the model includes a well-motivated cell-loss mechanism, apoptosis, which implies the existence of dormant (stationary) non-necrotic tumor states.

Following [1], we shall assume the tumor to be spherically symmetric and to occupy a region

$$
\{r<R(t)\} \quad\left(r=|x|, \quad x=\left(x_{1}, x_{2}, x_{3}\right)\right)
$$

at each time $t$; the boundary of the tumor is given by $r=R(t)$, an unknown function of $t$. Then, after nondimensionalization, the (dimensionless) nutrient concentration $\hat{\sigma}(r, t)$ will 
satisfy a reaction-diffusion equation of the form

$$
c \frac{\partial \hat{\sigma}}{\partial t}=\frac{1}{r^{2}} \frac{\partial}{\partial r}\left(r^{2} \frac{\partial \hat{\sigma}}{\partial r}\right)+\Gamma_{1}\left(\sigma_{B}-\hat{\sigma}\right)-\lambda_{0} \hat{\sigma}-\gamma_{1} \hat{\beta} \quad \text { if } \quad r<R(t), t>0
$$

where $\hat{\beta}$ is the (dimensionless) inhibitor concentration. Here the constants $\sigma_{B}$ and $\Gamma_{1}$ denote the (dimensionless) nutrient concentration in the vasculature and the rate of nutrient-in-bloodtissue transfer per unit length, respectively. Thus $\Gamma_{1}\left(\sigma_{B}-\sigma\right)$ accounts for the transfer of nutrient by means of the vasculature, whose presence stems from angiogenesis. The term $\lambda_{0} \hat{\sigma}$ is the nutrient consumption rate, $\gamma_{1} \hat{\beta}$ is the inhibitor consumption rate, and $c=T_{\text {diffusion }} / T_{\text {growth }}$ is the ratio of the nutrient diffusion time scale to the tumor growth (e.g. tumor doubling) time scale. Note that, typically, $T_{\text {diffusion }} \approx 1$ minute (see [8, pp. 194-195]) while $T_{\text {growth }} \approx 1$ day, so that $c \ll 1$.

The (dimensionless) inhibitor concentration $\hat{\beta}$ satisfies a similar reaction-diffusion equation $[1]$

$$
c_{2} \frac{\partial \hat{\beta}}{\partial t}=\frac{D_{2}}{r_{2}} \frac{\partial}{\partial r}\left(r^{2} \frac{\partial \hat{\beta}}{\partial r}\right)+\Gamma_{2}\left(\beta_{B}-\hat{\beta}\right)-\gamma_{2} \hat{\beta} \quad \text { if } \quad r<R(t), t>0
$$

where the constant $\beta_{B}$ denotes the (dimensionless) inhibitor concentration in the vasculature, $\Gamma_{2}$ is inhibitor-in-blood-tissue transfer per unit length, and $\gamma_{2} \hat{\beta}$ is the inhibitor consumption rate. Here $D_{2}$ is the (dimensionless) diffusion coefficient of the inhibitor concentration, and $c_{2} / D_{2}$ is the quotient of the inhibitor diffusion time scale to the tumor growth time scale; typically $D_{2} \sim 1$ so that $c_{2} \ll 1$. Actually $\Gamma_{2}=0$ in [1]; however if the inhibitor is partially fed through the vasculature, then $\Gamma_{2}>0$.

Note that in (1.1), (1.2)

$$
\frac{1}{r^{2}} \frac{\partial}{\partial r} r^{2} \frac{\partial}{\partial r}
$$

is meant to denote the radial part of the Laplace operator (in 3 dimensions), and therefore one must include the conditions

$$
\frac{\partial \hat{\sigma}}{\partial r}(0, t)=0, \quad \frac{\partial \hat{\beta}}{\partial r}(0, t)=0 .
$$

Assuming that the mass density of cells is constant, the principle of conservation of mass coincides with the principle of conservation of volume. A reasonable simplified approach to this principle, developed in [2], gives the relation

$$
\frac{d}{d t}\left(\frac{4}{3} \pi R^{3}(t)\right)=\int_{0}^{2 \pi} \int_{0}^{\pi} \int_{0}^{R(t)} S(\hat{\sigma}, \hat{\beta}) r^{2} \sin \theta d r d \theta d \varphi
$$

where $S(\hat{\sigma}, \hat{\beta})$ denotes the cell proliferation rate within the tumor. For simplicity we restrict ourselves to the inhibitor-free proliferation rate [1]

$$
S(\sigma)=\mu(\hat{\sigma}-\tilde{\sigma})
$$

where $\mu$ and $\tilde{\sigma}$ are positive constants. This means that the cell birth-rate is $\mu \hat{\sigma}$ while the death-rate (apoptosis) is given by $\mu \tilde{\widetilde{\sigma}}$. Finally, the external nutrient concentration is assumed 
to be a constant $\overline{\bar{\sigma}}$ and the external inhibitor concentration is assumed to be a constant $\overline{\bar{\beta}}$, so that

$$
\hat{\sigma}=\overline{\bar{\sigma}}, \quad \hat{\beta}=\overline{\bar{\beta}} \quad \text { on } \quad r=R(t) .
$$

The case where inhibitors are absent was recently studied by Friedman and Reitich [9] under the assumption that

$$
\overline{\bar{\sigma}}>\widetilde{\sigma}>\frac{\Gamma_{1} \sigma_{B}}{\Gamma_{1}+\lambda_{0}}
$$

Extending results obtained in [1] by perturbative and numerical studies, they proved by rigorous mathematical analysis that there exists a unique stationary solution $R(t) \equiv R_{s}$ and that this solution is asymptotically stable for the time-dependent problem, provided $c$ is sufficiently small; the asymptotic stability was only formally proved in [1] and only so in the limit case $c=0$.

The present work includes the presence of inhibitor, and our interest is to study the effect of the inhibitor on the tumor's growth. We shall not make the assumption (1.7), but for simplicity we shall require that

$$
\overline{\bar{\beta}}>\frac{\Gamma_{2} \beta_{B}}{\Gamma_{2}+\gamma_{2}}
$$

or, equivalently, that

$$
\Gamma_{2}\left(\beta_{B}-\overline{\bar{\beta}}\right)<\gamma_{2} \overline{\bar{\beta}}
$$

This means that the level of transfer rate of the inhibitor (by means of the vasculature) at the tumor's boundary is smaller than the inhibitor consumption rate at the boundary. We note that in [1] the term $\Gamma_{2}\left(\beta_{B}-\hat{\beta}\right)$ does not appear at all and so (1.8) is automatically satisfied.

REMARK 1.1. The methods presented in this paper can be extended to the case where (1.8) is not satisfied, but the results will be different.

It will be convenient to simplify the system (1.1)-(1.5) by introducing the following notation:

$$
\begin{aligned}
& c^{\prime}=\frac{c_{2}}{D_{2}}, \quad \gamma=\frac{\Gamma_{2}+\gamma_{2}}{D_{2}}, \quad \lambda=\Gamma_{1}+\lambda_{0}, \\
& \hat{\sigma}_{B}=\sigma_{B}-\frac{\gamma_{1}}{\Gamma_{1}} \cdot \frac{\Gamma_{2} \beta_{B}}{\Gamma_{2}+\gamma_{2}}, \quad \bar{\sigma}=\overline{\bar{\sigma}}-\frac{\Gamma_{1} \hat{\sigma}_{B}}{\Gamma_{1}+\lambda_{0}}, \quad \tilde{\sigma}=\tilde{\sigma}-\frac{\Gamma_{1} \hat{\sigma}_{B}}{\Gamma_{1}+\lambda_{0}} \\
& \bar{\beta}=\gamma_{1}\left(\bar{\beta}-\frac{\Gamma_{2} \beta_{B}}{\Gamma_{2}+\gamma_{2}}\right) .
\end{aligned}
$$

The specific value of $\mu$ (in (1.5)) will not affect the results of this paper. In order to slightly simplify the calculations, we shall take $\mu=3$. Then, introducing the normalized nutrient and inhibitor concentrations, $\sigma$ and $\beta$, by

$$
\sigma=\hat{\sigma}-\frac{\Gamma_{1} \sigma_{B}}{\Gamma_{1}+\lambda_{0}}, \quad \beta=\gamma_{1}\left(\hat{\beta}-\frac{\Gamma_{2} \beta_{B}}{\Gamma_{2}+\gamma_{2}}\right),
$$

the system (1.1)-(1.6) reduces to

$$
\begin{array}{ll}
c \frac{\partial \sigma}{\partial t}=\frac{1}{r^{2}} \frac{\partial}{\partial r}\left(r^{2} \frac{\partial \sigma}{\partial r}\right)-\lambda \sigma-\beta & \text { if } \quad r<R(t), t>0, \\
c^{\prime} \frac{\partial \beta}{\partial t}=\frac{1}{r^{2}} \frac{\partial}{\partial r}\left(r^{2} \frac{\partial \beta}{\partial t}\right)-\gamma \beta \quad \text { if } \quad r<R(t), t>0
\end{array}
$$




$$
\begin{aligned}
& \frac{\partial \sigma}{\partial r}(0, t)=0, \quad \sigma(R(t), t)=\bar{\sigma} \quad \text { if } \quad t>0, \\
& \frac{\partial \beta}{\partial r}(0, t)=0, \quad \beta(R(t), t)=\bar{\beta} \quad \text { if } \quad t>0, \\
& \frac{d R(t)}{d t}=\frac{3}{R^{2}(t)} \int_{0}^{R(t)}(\sigma(r, t)-\tilde{\sigma}) r^{2} d r \quad \text { if } \quad t>0 .
\end{aligned}
$$

Note that the assumption (1.8) means that

$$
\bar{\beta}>0 ;
$$

as mentioned above, we can actually allow $\bar{\beta}$ to be any real number though we shall not do so in this paper.

Finally we have initial conditions

$$
\begin{aligned}
& \sigma(r, 0)=\varphi_{0}(r), \quad \beta(r, 0)=\psi_{0}(r), \quad \text { if } \quad 0 \leq r \leq R_{0}, \\
& \frac{\partial \varphi_{0}}{\partial r}(0)=\frac{\partial \psi_{0}}{\partial r}(0)=0, \quad \text { and } \quad R(0)=R_{0}
\end{aligned}
$$

where $\varphi_{0}$ and $\psi_{0}$ are continuously differentiable functions.

We may view $\lambda$ and $\gamma$ as the consumption-transfer coefficients of the nutrient and inhibitor, respectively, and $\bar{\beta}$ as the normalized external concentration of the inhibitor.

In this paper we shall prove that there exists a unique solution to the system (1.12)-(1.18) for all $t>0$. However our main interest is to establish the existence of stationary solutions and to study their asymptotic stability with respect to the non-stationary solution. Our results will depend on just four constants:

$$
\begin{array}{ll}
\Lambda_{0}=\frac{1}{3} \frac{\tilde{\sigma}}{\bar{\sigma}}, & \Lambda_{1}=\frac{\bar{\beta}}{(\gamma-\lambda) \bar{\sigma}}, \\
\phi=\sqrt{\frac{\gamma}{\lambda}}, & \sigma_{0}=-\frac{\bar{\beta}}{\lambda} .
\end{array}
$$

We shall always assume that

$$
\begin{aligned}
& \min \left(\bar{\sigma}, \sigma_{0}\right) \leq \varphi_{0}(r) \leq \max (\bar{\sigma}, 0) \\
& 0 \leq \psi_{0}(r) \leq \bar{\beta} \quad \text { for } \quad 0 \leq r \leq R_{0} .
\end{aligned}
$$

For simplicity we always assume that

$$
\gamma \neq \lambda
$$

so that $\Lambda_{1}$ is well defined, and $\Lambda_{1} \neq 0$; the case $\gamma=\lambda$ is briefly considered in Remark 6.3 at the end of $\S 6$.

We first show that if $\tilde{\sigma}<\min \left(\bar{\sigma}, \sigma_{0}\right)$ then $R(t) \rightarrow \infty$ as $t \rightarrow \infty$, whereas if $\tilde{\sigma}>\max (\bar{\sigma}, 0)$ then $R(t) \rightarrow 0$ as $t \rightarrow \infty$. The function

$$
f(\eta)=\left(1-\Lambda_{1}\right) p(\eta)+\Lambda_{1} p(\phi \eta) \quad\left(p(\eta)=\frac{\eta \operatorname{coth} \eta-1}{\eta^{2}}\right)
$$

will play a fundamental role in studying all the remaining cases. 
We shall prove the following results:

$\left(\mathrm{A}_{1}\right)$ If $0<\Lambda_{0}<\frac{1}{3}$ then there exists a unique stationary solution $\left(\sigma_{s}, \beta_{s}, R_{s}\right)$ which is asymptotically stable if $\bar{\sigma} \geq 0$ and unstable if $\bar{\sigma}<0$, with respect to the time-dependent solutions of $(1.12)-(1.18)$.

$\left(\mathrm{A}_{2}\right)$ If $-\frac{1}{\phi+1} \leq(\phi-1) \Lambda_{1} \leq \phi$, and $\Lambda_{0} \notin\left(0, \frac{1}{3}\right)$ then no stationary solutions exist.

$\left(\mathrm{A}_{3}\right)$ If $(\phi-1) \Lambda_{1}>\phi$ then $\Lambda_{0}^{*}=\min _{\eta>0} f(\eta)<0$, and when $\Lambda_{0} \geq \frac{1}{3}$, or $\Lambda_{0} \leq \Lambda_{0}^{*}$, no stationary solutions exist. However when $\Lambda_{0}^{*}<\Lambda_{0}<0$ then there exist two stationary solutions, $\left(\sigma_{s}^{-}, \beta_{s}^{-}, R_{s}^{-}\right)$and $\left(\sigma_{s}^{+}, \beta_{s}^{+}, R_{s}^{+}\right)$, with $R_{s}^{-}<R_{s}^{+} ; R_{s}^{-}$is asymptotically stable whereas $R_{s}^{+}$is unstable.

$\left(\mathrm{A}_{4}\right)$ If $(\phi-1) \Lambda_{1}<-\frac{1}{\phi+1}$ then $\Lambda_{0}^{* *}=\max _{\eta>0} f(\eta)>0$, and when $\Lambda_{0} \leq 0$, or $\Lambda_{0} \geq \Lambda_{0}^{* *}$, no stationary solution exist. However when $\frac{1}{3}<\Lambda_{0}<\Lambda_{0}^{* *}$ then there exist two stationary solutions as in case $\left(\mathrm{A}_{3}\right)$, and again $R_{s}^{-}$is asymptotically stable whereas $R_{s}^{+}$is unstable.

The above results are proved under the assumption that $c$ and $c^{\prime}$ are sufficiently small. We shall also prove that when no stationary solutions exist then there are initial data for which

$$
\lim _{t \rightarrow \infty} R(t)=\infty
$$

The mathematical results of this paper have implications for the treatment of tumor. Consider for example the case where $\tilde{\sigma}<0<\bar{\sigma}$, so that with no inhibitors the tumor will grow unboundedly. Then, by administering inhibitor (drug) with sufficiently large external (normalized) concentration $\bar{\beta}$ we are able to contain the tumor. In fact, there are two critical parameters, $\beta^{*}$ and $\beta^{* *}$, with $\beta^{*}<\beta^{* *}$, where $\beta^{*}$ does not depend on the initial conditions and $\beta^{* *}$ depends on the initial conditions, such that the following holds: If $\bar{\beta}>\beta^{*}$ then a dormant situation can be achieved provided the initial size of the tumor is below a certain radius $R_{s}^{+}\left(\beta^{*}\right)$, whereas if $\bar{\beta}$ is further increased so that it becomes larger than $\beta^{* *}$ then the tumor will definitely be contained and it will evolve into a dormant one with radius $R_{s}^{-}$; more details will be given in $\S 7$.

\section{Global existence and uniqueness.}

THEOREM 2.1. The system (1.12)-(1.18) has a unique solution $(\sigma, \beta, R)$ for all $t>0$, and

$$
\begin{aligned}
& 0<\beta(r, t)<\bar{\beta} \quad \text { for } \quad 0 \leq r<R(t), \quad t>0, \\
& \min \left(\bar{\sigma}, \sigma_{0}\right)<\sigma(r, t)<\max (\bar{\sigma}, 0) \quad \text { for } \quad 0 \leq r<R(t) \quad t>0, \\
& R_{0} \exp \left\{t\left[\min \left(\bar{\sigma}, \sigma_{0}\right)-\tilde{\sigma}\right]\right\} \leq R(t) \leq R_{0} \exp \{t[\max (\bar{\sigma}, 0)-\tilde{\sigma}]\} \quad \text { for } \quad t \geq 0, \\
& {\left[\min \left(\bar{\sigma}, \sigma_{0}\right)-\tilde{\sigma}\right] \leq \frac{\dot{R}(t)}{R(t)} \leq[\max (\bar{\sigma}, 0)-\tilde{\sigma}] \quad \text { for } \quad t \geq 0 .}
\end{aligned}
$$

Proof. Local existence and uniqueness can be proved as in the case of the Stefan problem [1, Chap. 8]. If we can prove the a priori bounds (2.3), (2.4), then the solution can be continued for all $t>0$. On the other hand (2.4), and consequently also (2.3), follows from (2.2) and (1.16). Thus it remains to prove $(2.2)$ and (2.1). These assertions follow by the maximum principle. Indeed, (2.1) is rather immediate; as for (2.2), by comparison we have that $\sigma_{1}(r, t) \leq \sigma(r, t) \leq$ $\sigma_{2}(r, t)$, where

$$
\begin{aligned}
& c \frac{\partial \sigma_{1}}{\partial t}=\frac{1}{r^{2}} \frac{\partial}{\partial r}\left(r^{2} \frac{\partial \sigma_{1}}{\partial r}\right)-\lambda \sigma_{1}-\bar{\beta} \\
& c \frac{\partial \sigma_{2}}{\partial t}=\frac{1}{r^{2}} \frac{\partial}{\partial r}\left(r^{2} \frac{\partial \sigma_{2}}{\partial r}\right)-\gamma \sigma_{2}
\end{aligned}
$$


for $0<r<R(t), t>0$ and

$$
\begin{aligned}
& \sigma_{1}(R(t), t)=\sigma_{2}(R(t), t)=\bar{\sigma} \quad \text { if } \quad t>0, \\
& \sigma_{1}(r, 0) \equiv \min \left(\bar{\sigma}, \sigma_{0}\right), \quad \sigma_{2}(r, 0) \equiv \max (\bar{\sigma}, 0) \quad \text { if } \quad 0 \leq r<R(0) .
\end{aligned}
$$

By comparison

$$
\sigma_{2}(r, t) \leq \max (\bar{\sigma}, 0)
$$

and also (using the relation $\lambda \sigma_{0}+\bar{\beta}=0$ )

$$
\sigma_{1}(r, t) \geq \min \left(\bar{\sigma}, \sigma_{0}\right)
$$

so that (2.2) holds.

From (2.3) we get:

Corollary 2.2. (i) If $\max (\bar{\sigma}, 0)<\tilde{\sigma}$ then $\lim _{t \rightarrow \infty} R(t)=0$; (ii) If $\min \left(\bar{\sigma}, \sigma_{0}\right)>\tilde{\sigma}$ then $\lim _{t \rightarrow \infty} R(t)=\infty$.

Therefore in studying the asymptotic behavior of $R(t)$ we shall concentrate just on the case where

$$
\min \left(\bar{\sigma}, \sigma_{0}\right) \leq \tilde{\sigma} \leq \max (\bar{\sigma}, 0)
$$

Henceforth it will be assumed that (2.7) is satisfied.

3. Steady state solutions. The steady state solution, if existing, is determined by the system

$$
\begin{aligned}
& \frac{1}{r^{2}} \frac{\partial}{\partial r}\left(r^{2} \frac{\partial \sigma_{s}}{\partial r}\right)-\lambda \sigma_{s}-\beta_{s}=0, \quad 0<r<R_{s} \\
& \frac{1}{r^{2}} \frac{\partial}{\partial r}\left(r^{2} \frac{\partial \beta_{s}}{\partial r}\right)-\gamma \beta_{s}=0, \quad 0<r<R_{s} \\
& \frac{\partial \sigma_{s}}{\partial r}(0)=0, \quad \sigma_{s}\left(R_{s}\right)=\bar{\sigma}, \\
& \frac{\partial \beta_{s}}{\partial r}(0)=0, \quad \beta_{s}\left(R_{s}\right)=\bar{\beta},
\end{aligned}
$$

and the equation

$$
\frac{3}{R_{s}^{2}} \int_{0}^{R_{s}} \sigma(r) r^{2} d r=\tilde{\sigma} R_{s}
$$

For a given $R_{s}>0$, the solution of (3.1)-(3.4) is

$$
\begin{aligned}
\beta_{s} & =\frac{\bar{\beta} R_{s}}{\sinh \left(\sqrt{\gamma} R_{s}\right)} \frac{\sinh (\sqrt{\gamma} r)}{r}, \\
\sigma_{s} & =\left(1-\Lambda_{1}\right) \frac{\bar{\sigma} R_{s}}{\sinh \left(\sqrt{\lambda} R_{s}\right)} \frac{\sinh (\sqrt{\lambda} r)}{r}+\Lambda_{1} \frac{\bar{\sigma} R_{s}}{\sinh \left(\sqrt{\gamma} R_{s}\right)} \frac{\sinh (\sqrt{\gamma} r)}{r} .
\end{aligned}
$$

Substituting (3.6), (3.7) into (3.5) we obtain

$$
\left(1-\Lambda_{1}\right) \bar{\sigma} \frac{\sqrt{\lambda} R_{s} \operatorname{coth}\left(\sqrt{\lambda} R_{s}\right)-1}{\lambda R_{s}}+\Lambda_{1} \bar{\sigma} \frac{\sqrt{\gamma} R_{s} \operatorname{coth}\left(\sqrt{\gamma} R_{s}\right)-1}{\gamma R_{s}}=\frac{1}{3} \tilde{\sigma} R_{s}
$$


this equation was derived in [1].

It will be convenient to introduce the variable

$$
\eta_{s}=\sqrt{\lambda} R_{s}
$$

and the functions

$$
\begin{aligned}
& p(\eta)=\frac{\eta \operatorname{coth} \eta-1}{\eta^{2}}, \\
& f(\eta)=\left(1-\Lambda_{1}\right) p(\eta)+\Lambda_{1} p(\phi \eta) .
\end{aligned}
$$

Dividing (3.8) by $\bar{\sigma} R_{s}$, we obtain the relation

$$
f\left(\eta_{s}\right)=\Lambda_{0}
$$

Thus we have proved:

LEMMA 3.1. The system (3.1)-(3.5) has a solution if and only if equation (3.12) has a positive solution $\eta_{s}$, and, in that case, the solution $R_{s}, \sigma_{s}, \beta_{s}$ is given by (3.9), (3.7) and (3.6).

The remaining of this section is devoted to determining how many solutions equation (3.12) admits. We first need two lemmas.

LEMMA 3.2. (i) $p^{\prime}(\eta)<0$ for all $\eta>0$;

(ii) $\lim _{\eta \rightarrow 0} p(\eta)=\frac{1}{3}, \lim _{\eta \rightarrow \infty} p(\eta)=0, \lim _{\eta \rightarrow \infty} \eta p(\eta)=1$;

(iii) $0<p(\eta)<\frac{1}{3}, 0<\eta p(\eta)<1$ for all $\eta>0$.

The first part is proved in [9], and the other parts are rather immediate.

LEMMA 3.3. The function

$$
k(\eta)=\frac{\eta p^{\prime \prime}(\eta)}{p^{\prime}(\eta)}
$$

is strictly monotone decreasing and in fact, $k^{\prime}(\eta)<0$ for all $\eta>0$.

Proof. By direct computation

$$
k(\eta)=\frac{2\left(\sinh ^{3} \eta-\eta^{3} \cosh \eta\right)}{\left[\eta^{2}+\eta \cosh \eta \sinh \eta-2 \sinh ^{2} \eta\right] \sinh \eta}-2
$$

and

where

$$
k^{\prime}(\eta)=-\frac{2 g(\eta)}{\left[\eta^{2}+\eta \cosh \eta \sinh \eta-2 \sinh ^{2} \eta\right]^{2} \sinh ^{2} \eta}
$$

$$
\begin{aligned}
g(\eta)= & \sinh ^{5} \eta \cosh \eta+\eta \sinh ^{4} \eta+6 \eta^{3} \sinh ^{2} \eta \cosh ^{2} \eta+2 \eta^{3} \sinh ^{2} \eta \\
& +\eta^{4} \sinh \eta \cosh \eta-8 \eta^{2} \sinh ^{3} \eta \cosh \eta-2 \eta^{4} \sinh \eta \cosh ^{3} \eta-\eta^{5}
\end{aligned}
$$

Thus it remains to show that $g(\eta)>0$ for all $\eta>0$.

Writing

$$
\begin{aligned}
g(\eta) e^{-6 \eta}= & \frac{1}{64}-\left(\frac{1}{16}-\frac{1}{16} \eta+\frac{1}{2} \eta^{2}-\frac{3}{8} \eta^{3}+\frac{1}{8} \eta^{4}\right) e^{-2 \eta}+\left(\frac{5}{64}-\frac{1}{4} \eta+\eta^{2}+\frac{1}{2} \eta^{3}\right) e^{-4 \eta} \\
& +\left(\frac{3}{8} \eta-\frac{7}{4} \eta^{3}-\eta^{5}\right) e^{-6 \eta}+\left(-\frac{5}{64}-\frac{1}{4} \eta-\eta^{2}+\frac{1}{2} \eta^{3}\right) e^{-8 \eta} \\
& +\left(\frac{1}{16}+\frac{1}{16} \eta+\frac{1}{2} \eta^{2}+\frac{3}{8} \eta^{3}+\frac{1}{8} \eta^{4}\right) e^{-10 \eta}-\frac{1}{64} e^{-12 \eta}
\end{aligned}
$$


one can easily verify that, for $\eta \geq 2$,

$$
g(\eta) e^{-6 \eta} \geq \frac{1}{64}-\left(\frac{1}{16}-\frac{1}{16} \eta+\frac{1}{2} \eta^{2}-\frac{3}{8} \eta^{3}+\frac{1}{8} \eta^{4}\right) e^{-2 \eta}-\eta^{5} e^{-6 \eta} \equiv h(\eta) .
$$

But, as easily seen, the function $\eta^{5} e^{-6 \eta}$ is monotone decreasing if $\eta \geq \frac{5}{6}$ and the function

$$
\left(\frac{1}{16}-\frac{1}{16} \eta+\frac{1}{2} \eta^{2}-\frac{3}{8} \eta^{3}+\frac{1}{8} \eta^{4}\right) e^{-2 \eta}
$$

is monotone decreasing for $\eta \geq 2$. Since also $h(2.25)=1.57163 \times 10^{-4}>0$, it follows that $g(\eta)>0$ if $\eta \geq 2.25$.

For $\eta<2.25$ we write

$$
\begin{aligned}
& \sum_{k=0}^{7} \frac{\eta^{2 k}}{(2 k+1) !}<\frac{\sinh \eta}{\eta}<\sum_{k=0}^{7} \frac{\eta^{2 k}}{(2 k+1) !}+\frac{\eta^{16}}{17 !} \cosh (2.25) \\
& \sum_{k=0}^{7} \frac{\eta^{2 k}}{(2 k) !}<\cosh \eta<\sum_{k=0}^{7} \frac{\eta^{2 k}}{(2 k) !}+\frac{\eta^{16}}{17 !} \cosh (2.25)
\end{aligned}
$$

Applying the lower bounds to the five positive terms in $g(\eta) / \eta^{5}$ and the upper bounds to the first two negative terms in $g(\eta)$, we obtain after some calculations,

$$
\begin{aligned}
\frac{g(\eta)}{\eta^{5}}>[ & 11+\frac{34}{3} \eta^{2}+\frac{82}{15} \eta^{4}+\frac{292}{189} \eta^{6}+\frac{4058}{14175} \eta^{8}+\frac{17692}{467775} \eta^{10} \\
& \left.+\frac{62492}{16372125} \eta^{12}+\frac{198944}{638512875} \eta^{14}\right]-\left[11+\frac{34}{3} \eta^{2}+\frac{82}{15} \eta^{4}+\frac{292}{189} \eta^{6}\right. \\
& \left.+\frac{4058}{14175} \eta^{8}+\frac{1156}{31185} \eta^{10}+\frac{2132}{606375} \eta^{12}+\frac{2944}{11609325} \eta^{14}+\ell(\eta) \eta^{16}\right]
\end{aligned}
$$

where $\ell(\eta)$ is a polynomial of degree 48 with positive coefficients, so that

$$
\ell(\eta)<\ell(2.25)=1.83644 \times 10^{-5} \quad \text { if } \quad 0<\eta<2.25 .
$$

For such $\eta$ we then get, by computing the coefficients of each power of $\eta$ in the above expression,

$$
\begin{aligned}
\frac{g(\eta)}{\eta^{15}} & >\frac{32}{42525}+\frac{64}{212625} \eta^{2}+\frac{2848}{49116375} \eta^{4}-1.83644 \times 10^{-5} \eta^{6} \\
& =10^{-5}\left[75.2499+30.0999 \eta^{2}+\left(5.79847-1.83644 \eta^{2}\right) \eta^{4}\right]
\end{aligned}
$$

and the expression in parenthesis is $>-3.4985$ if $0<\eta<2.25$. Hence

$$
\frac{g(\eta)}{\eta^{15}} 10^{5} \geq 75.2499+\left(30.0999-3.4985 \eta^{2}\right) \eta^{2}>0
$$

if $0<\eta<2.25$ since the expression in parenthesis is positive.

Corollary 3.4. If $\phi>1(0<\phi<1)$ then

$$
\frac{d}{d \eta} \frac{p^{\prime}(\phi \eta)}{p^{\prime}(\eta)}<0 \quad(>0) \quad \text { for all } \quad \eta>0
$$


Proof. Clearly

$$
\frac{d}{d \eta} \frac{p^{\prime}(\phi \eta)}{p^{\prime}(\eta)}=\frac{p^{\prime}(\eta) \cdot \phi p^{\prime \prime}(\phi \eta)-p^{\prime}(\phi \eta) \cdot p^{\prime \prime}(\eta)}{\left(p^{\prime}(\eta)\right)^{2}}=\left(\frac{\phi \eta p^{\prime \prime}(\phi \eta)}{p^{\prime}(\phi \eta)}-\frac{\eta p^{\prime \prime}(\eta)}{p^{\prime}(\eta)}\right) \cdot \frac{p^{\prime}(\phi \eta)}{\eta p^{\prime}(\eta)}
$$

Since the last factor is positive by Lemma 3.2, the assertion follows from Lemma 3.3.

In the next theorem we analyze the behavior of the function $f(\eta)$ defined in (3.11); note that

$$
f(0)=\frac{1}{3}, \quad \lim _{\eta \rightarrow \infty} f(\eta)=0 .
$$

THEOREM 3.5. (i) If $-\frac{1}{\phi+1}<(\phi-1) \Lambda_{1} \leq \phi$ then

$$
f^{\prime}(\eta)<0 \quad \text { for all } \quad \eta>0 .
$$

(ii) If $(\phi-1) \Lambda_{1}>\phi$ then there is a unique $\eta_{0}$ such that

$$
f^{\prime}(\eta)<0 \quad \text { for } 0<\eta<\eta_{0}, \quad f^{\prime}(\eta)>0 \quad \text { for } \quad \eta>\eta_{0}
$$

and, by (3.14),

$$
\Lambda_{0}^{*}=f\left(\eta_{0}\right)=\min _{\eta>0} f(\eta)<0
$$

(iii) If $(\phi-1) \Lambda_{1}<-\frac{1}{\phi+1}$ then there exists a unique $\eta_{0}>0$ such that

$$
f^{\prime}(\eta)>0 \quad \text { for } 0<\eta<\eta_{0}, \quad f^{\prime}(\eta)<0 \quad \text { for } \quad \eta>\eta_{0}
$$

and, by (3.14),

$$
\Lambda_{0}^{* *}=f\left(\eta_{0}\right)=\max _{\eta>0} f(\eta)>\frac{1}{3}
$$

Proof. Note that

$$
f^{\prime}(\eta)=p^{\prime}(\eta)\left[\left(1-\Lambda_{1}\right)+\phi \Lambda_{1} \frac{p^{\prime}(\phi \eta)}{p^{\prime}(\eta)}\right]
$$

Also, by simple calculation,

$$
\lim _{\eta \rightarrow 0} \frac{p^{\prime}(\phi \eta)}{p^{\prime}(\eta)}=\phi, \quad \lim _{\eta \rightarrow \infty} \frac{p^{\prime}(\phi \eta)}{p^{\prime}(\eta)}=\frac{1}{\phi^{2}} .
$$

Consider first the case $0 \leq(\phi-1) \Lambda_{1} \leq \phi$. Then $\Lambda_{1} \geq 0$ (respectively, $\left.\Lambda_{1}<0\right)$ implies $\phi \geq 1$ (respectively, $\phi<1$ ), so that, by Corollary 3.4, $p^{\prime}(\phi \eta) / p^{\prime}(\eta)$ is strictly monotone decreasing (respectively, increasing). Consequently, by (3.19),

$$
\frac{p^{\prime}(\phi \eta)}{p^{\prime}(\eta)}>\frac{1}{\phi^{2}} \quad\left(\text { respectively }, \quad<\frac{1}{\phi^{2}}\right)
$$

so that the expression in brackets in $(3.18)$ is $>\left[\left(1-\Lambda_{1}\right)+\phi \Lambda_{1} / \phi^{2}\right]$. Since $p^{\prime}(\eta)<0$, we get from (3.18),

$$
f^{\prime}(\eta)<p^{\prime}(\eta)\left[\left(1-\Lambda_{1}\right)+\frac{\phi \Lambda_{1}}{\phi^{2}}\right]=p^{\prime}(\eta) \frac{\phi-(\phi-1) \Lambda_{1}}{\phi} \leq 0
$$


and (3.15) follows. The remaining case of $(\mathrm{i})$, namely, $-\frac{1}{\phi+1} \leq(\phi-1) \Lambda_{1}<0$, can be proved similarly.

To prove (ii) note that $(\phi-1) \Lambda_{1}>\phi$ implies that

$$
\begin{array}{lll}
\frac{1}{\phi^{2}}<\frac{\Lambda_{1}-1}{\phi \Lambda_{1}}<\phi & \text { if } \quad \Lambda_{1}>0 & (\Longleftrightarrow \quad \phi>1), \\
\phi<\frac{\Lambda_{1}-1}{\phi \Lambda_{1}}<\frac{1}{\phi^{2}} & \text { if } \quad \Lambda_{1}<0 & (\Longleftrightarrow \quad \phi<1) .
\end{array}
$$

From Lemma 3.2(i) and (3.18), (3.19), (3.20) we see that if $\Lambda_{1}>0$ then

$$
\begin{array}{ll}
f^{\prime}(\eta) \sim p^{\prime}(\eta)\left[\left(1-\Lambda_{1}\right)+\Lambda_{1} \phi^{2}\right]<0 & \text { if } \quad \eta \quad \text { is near } 0 \\
f^{\prime}(\eta) \sim p^{\prime}(\eta)\left[\left(1-\Lambda_{1}\right)+\frac{\Lambda_{1}}{\phi}\right]>0 \quad \text { if } \quad \eta \quad \text { is near } \infty .
\end{array}
$$

Similarly if $\Lambda_{1}<0$ then (using (3.21))

$$
\begin{array}{cc}
f^{\prime}(\eta)<0 \quad \text { if } \quad \eta \text { is near } 0, \\
f^{\prime}(\eta)>0 \quad \text { if } \quad \eta \text { is near } \infty .
\end{array}
$$

Hence in both cases there exists a point $\eta_{0}$ such that $f^{\prime}\left(\eta_{0}\right)=0$. Since $p^{\prime}(\eta)$ never vanishes whereas the expression in brackets in (3.18) has everywhere negative derivative if $\phi>1$ and everywhere positive derivative if $\phi<1$ (by Corollary 3.4), we deduce that $f^{\prime}(\eta)$ has a unique zero and (ii) readily follows. Finally, (iii) is established by the same argument as (ii).

From Lemma 3.1 and Theorem 3.5 we obtain the following results concerning the existence of stationary solution of (3.1)-(3.5):

THEOREM 3.6. (i) If $-\frac{1}{\phi+1}<(\phi-1) \Lambda_{1} \leq \phi$, then for $0<\Lambda_{0}<\frac{1}{3}$ there exists a unique stationary solution $\left(\sigma_{s}, \beta_{s}, R_{s}\right)$, while for $\Lambda_{0} \notin\left(0, \frac{1}{3}\right)$ there are no stationary solutions.

(ii) If $(\phi-1) \Lambda_{1}>\phi$ then for $0 \leq \Lambda_{0}<\frac{1}{3}$ there exists a unique stationary solution $\left(\sigma_{s}, \beta_{s}, R_{s}\right)$; for $\Lambda_{0}^{*}<\Lambda_{0}<0 \quad\left(\Lambda_{0}^{*}=\min _{\eta>0} f(\eta)<0\right)$ there are two stationary solutions $\left(\sigma_{s}^{-}, \beta_{s}^{-}, R_{s}^{-}\right)$and $\left(\sigma_{s}^{+}, \beta_{s}^{+}, R_{s}^{+}\right)$with $R_{s}^{-}<R_{s}^{+} ;$for $\Lambda_{0}=\Lambda_{0}^{*}$ the two solutions coincide, and for $\Lambda_{0} \notin\left[\Lambda_{0}^{*}, \frac{1}{3}\right)$ there are no stationary solutions.

(iii) If $(\phi-1) \Lambda_{1}<-\frac{1}{\phi+1}$ then for $0<\Lambda_{0} \leq \frac{1}{3}$ there exists a unique stationary solution $\left(\sigma_{s}, \beta_{s}, R_{s}\right) ;$ for $\frac{1}{3}<\Lambda_{0}<\Lambda_{0}^{* *} \quad\left(\Lambda_{0}^{* *}=\max _{\eta>0} f(\eta)>\frac{1}{3}\right)$ there are two stationary solutions $\left(\sigma_{s}^{-}, \beta_{s}^{-}, R_{s}^{-}\right)$and $\left(\sigma_{s}^{+}, \beta_{s}^{+}, R_{s}^{+}\right)$with $R_{s}^{-}<R_{s}^{+} ;$for $\Lambda_{0}=\Lambda_{0}^{* *}$ the two solutions coincide, and for $\Lambda_{0} \notin\left(0, \Lambda_{0}^{* *}\right]$ there are no stationary solutions.

We shall be interested in the asymptotic behavior of the solution of (1.12)-(1.18). In order to gain some insight about what to expect, we briefly consider the limiting case where $c=c^{\prime}=0$. Then, for each $t>0$,

$$
\begin{aligned}
& \beta(r, t)=\frac{\bar{\beta} R(t)}{\sinh (\sqrt{\gamma} R(t))} \frac{\sinh (\sqrt{\gamma} r)}{r} \\
& \sigma(r, t)=\left(1-\Lambda_{1}\right) \frac{\bar{\sigma} R(t)}{\sinh (\sqrt{\lambda} R(t))} \frac{\sinh (\sqrt{\lambda} r)}{r}+\Lambda_{1} \frac{\bar{\sigma} R(t)}{\sinh (\sqrt{\gamma} R(t))} \frac{\sinh (\sqrt{\gamma} r)}{r} .
\end{aligned}
$$


Substituting the last expression into (1.16) and setting $\eta(t)=\sqrt{\lambda} R(t)$, we get

$$
\frac{d \eta}{d t}=\bar{\sigma} \eta\left[f(\eta)-\Lambda_{0}\right]
$$

By applying Theorems 3.5, 3.6 we easily obtain the following conclusions:

$\left(\mathrm{R}_{1}\right)$ If $0 \leq(\phi-1) \Lambda_{1} \leq \phi$ (which implies $\bar{\sigma} \geq 0$ ) then, for $0<\Lambda_{0}<\frac{1}{3}, \lim _{t \rightarrow \infty} R(t)=R_{s}$ for any initial radius $R_{0}$, that is, $\left(\sigma_{s}, \beta_{s}, R_{s}\right)$ is globally asymptotically stable (Figure 1 ); for $\Lambda_{0} \geq \frac{1}{3}$ we have $\lim _{t \rightarrow \infty} R(t)=0$, and for $\Lambda_{0} \leq 0$ we have $\lim _{t \rightarrow \infty} R(t)=\infty$.

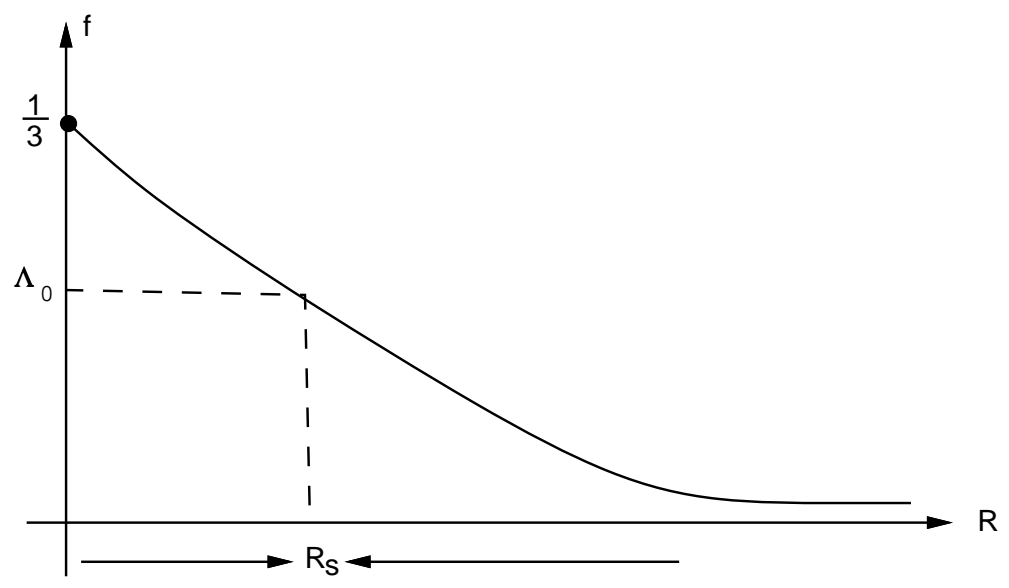

FIG. $1.0 \leq(\phi-1) \Lambda_{1} \leq \phi$.

$\left(\mathrm{R}_{2}\right)$ If $(\phi-1) \Lambda_{1}>\phi$ (which implies $\bar{\sigma}>0$ ) then for $0 \leq \Lambda_{0}<\frac{1}{3}$ the solution $\left(\sigma_{s}, \beta_{s}, R_{s}\right)$ is again asymptotically stable (Figure $2(\mathrm{a})$ ); for $\Lambda_{0}^{*}<\Lambda_{0}<0$ the solution $\left(\sigma_{s}^{-}, \beta_{s}^{-}, R_{s}^{-}\right.$) is locally asymptotically stable with the attraction region of $R_{s}^{-}$being $\left(0, R_{s}^{+}\right)$, and for any $R_{0}>R_{s}^{+}$we have $\lim _{t \rightarrow \infty} R(t)=\infty$ (Figure $2(\mathrm{~b})$ ). For $\Lambda_{0} \geq \frac{1}{3}$ we have $\lim _{t \rightarrow \infty} R(t)=0$, and for $\Lambda_{0}<\Lambda_{0}^{*}$ we have $\lim _{t \rightarrow \infty} R(t)=\infty$.

$\left(\mathrm{R}_{3}\right)$ If $-\frac{1}{\phi+1} \leq(\phi-1) \Lambda_{1}<0$ (which implies $\bar{\sigma}<0$ ) then, for $0<\Lambda_{0}<\frac{1}{3}$ we have: $\lim _{t \rightarrow \infty} R(t)=0$ if $R_{0}<R_{s}, \lim _{t \rightarrow \infty} R(t)=\infty$ if $R_{0}>R_{s}$, hence $\left(\sigma_{s}, \beta_{s}, R_{s}\right)$ is unstable (Figure 3); for $\Lambda_{0} \geq \frac{1}{3}$ we have $\lim _{t \rightarrow \infty} R(t)=\infty$ and for $\Lambda_{0} \leq 0$ we have $\lim _{t \rightarrow \infty} R(t)=0$.

$\left(\mathrm{R}_{4}\right)$ If $(\phi-1) \Lambda_{1}<-\frac{1}{\phi+1}$ (which implies $\bar{\sigma}<0$ ) then, for $0<\Lambda_{0} \leq \frac{1}{3}$ we have the same conclusion as in $\left(\mathrm{R}_{3}\right)$ (Figure $4(\mathrm{a})$ ); for $\frac{1}{3}<\Lambda_{0}<\Lambda_{0}^{* *}$ the solution $\left(\sigma_{s}^{-}, \beta_{s}^{-}, R_{s}^{-}\right)$is locally asymptotically stable with the domain of attraction of $R_{s}^{-}$being $\left(0, R_{s}^{+}\right)$, and for $R_{0}>R_{s}^{+}$we have $\lim _{t \rightarrow \infty} R(t)=\infty$ (Figure $4(\mathrm{~b})$ ). For $\Lambda_{0}>\Lambda_{0}^{* *}$ we have $\lim _{t \rightarrow \infty} R(t)=\infty$ and for $\Lambda_{0} \leq 0$ we have $\lim _{t \rightarrow \infty} R(t)=0$.

The rest of the paper is devoted to the extension of the above results to the non-degenerate case where $c>0, c^{\prime}>0$.

4. A non-extinction theorem. In cases $\left(\mathrm{R}_{1}\right),\left(\mathrm{R}_{2}\right),\left(\mathrm{R}_{4}(\mathrm{~b})\right)$ (see Figures $\left.1,2,4(\mathrm{~b})\right)$ we have $\lim _{t \rightarrow \infty} R(t)>0$, so that $R(t)$ does not become extinct as $t \rightarrow \infty$. In this section we shall extend this result to non-vanishing $c, c^{\prime}$, proving that $\liminf _{t \rightarrow \infty} R(t)>0$. Note that in cases $\left(\mathrm{R}_{1}\right)$ and $\left(\mathrm{R}_{2}\right), \bar{\sigma}>0, \tilde{\sigma}<\bar{\sigma}$, whereas in case $\left(\mathrm{R}_{4}(\mathrm{~b})\right) \tilde{\sigma}<\bar{\sigma}<0$. Since we always assume that (2.7) holds (recall Corollary 2.2), in all these three cases we have $\sigma_{0}<\tilde{\sigma}<\bar{\sigma}$. 


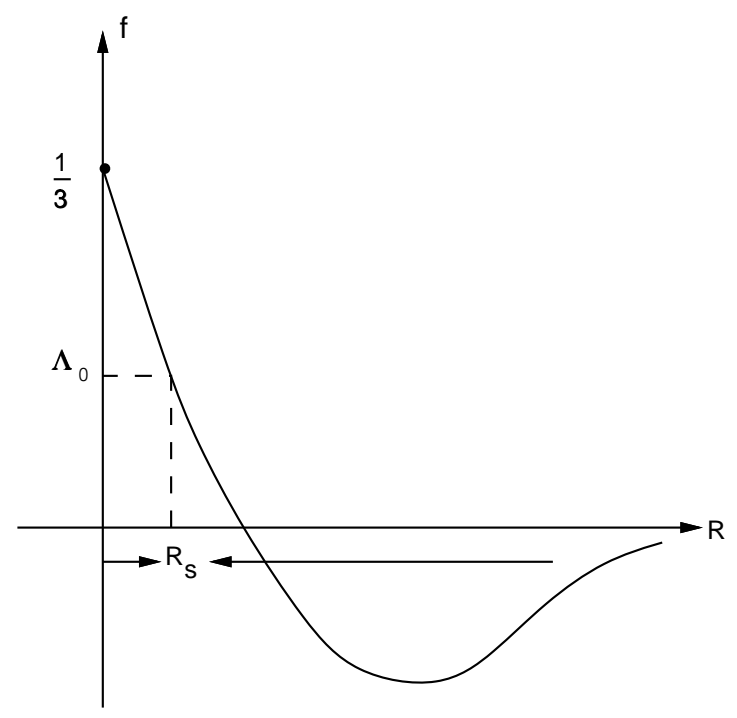

(a)

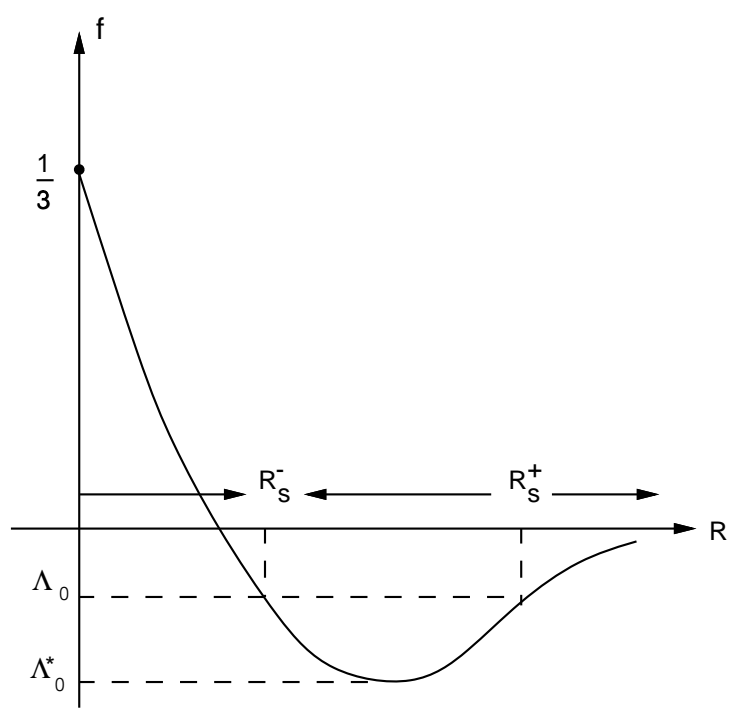

(b)

FIG. 2. $(\phi-1) \Lambda_{1}>\phi$.

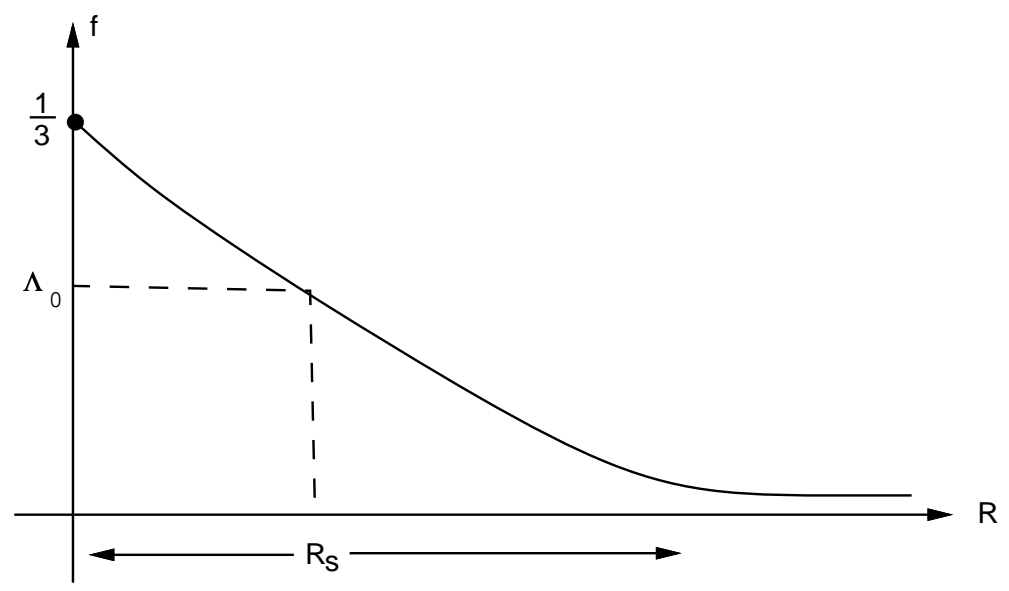

FIG. 3. $-\frac{1}{\phi+1} \leq(\phi-1) \Lambda_{1}<0$.

We now state the non-extinction result:

THEOREM 4.1. If $\sigma_{0}<\tilde{\sigma}<\bar{\sigma}$ then for any $\varepsilon>0$ there exist positive constants $\delta_{0}=\delta_{0}(\varepsilon)$ and $T_{0}=T_{0}\left(\varepsilon, R_{0}\right)$ such that, if $0<c \leq \varepsilon$, then

$$
R(t) \geq \delta_{0} \quad \text { for all } \quad t \geq T_{0}
$$

REMARK 4.1. It is important to note that $\delta_{0}$ is independent of $R_{0}$.

Proof. The proof is an extension and some simplification of the corresponding proof for the inhibitor-free case [9]. We choose $\sigma_{*}<0$ such that

$$
\lambda \sigma_{*}+\bar{\beta} \leq 0 \quad \text { and } \quad \bar{\sigma}_{2} \equiv \bar{\sigma}-\sigma_{*}>0
$$




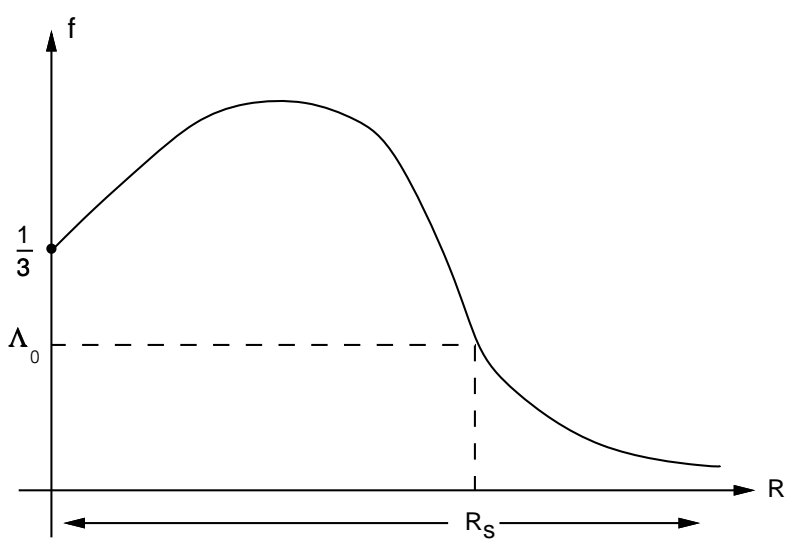

(a)

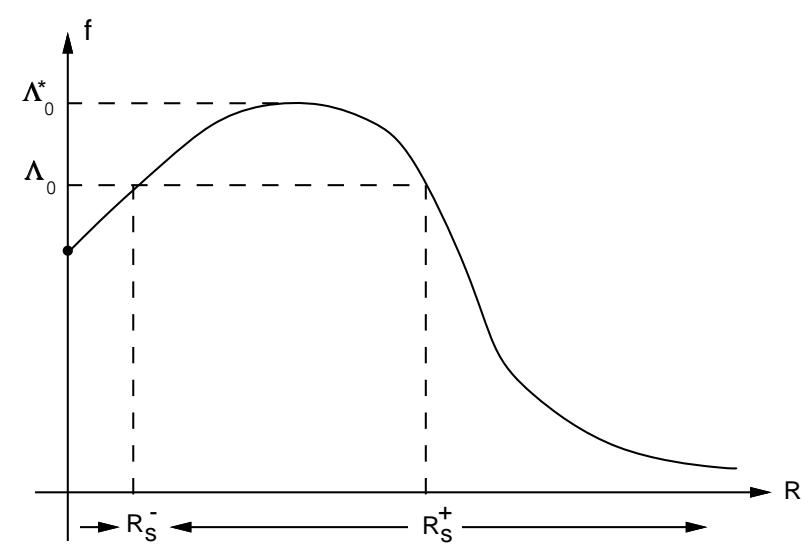

(b)

FIG. 4. $(\phi-1) \Lambda_{1}<-\frac{1}{\phi+1}$.

and introduce the function

$$
v(r, t)=\bar{v}(r, t)+\sigma_{*} \equiv \bar{\sigma}_{2} \frac{R(t)}{\sinh (M R(t))} \frac{\sinh (M r)}{r}+\sigma_{*} .
$$

Note that

$$
v_{t}=-\bar{v} \frac{\dot{R}(t)}{R(t)}[M R(t) \operatorname{coth}(M R(t))-1]
$$

and, since $\lambda \sigma_{*}+\bar{\beta} \leq 0$

$$
c v_{t}-\Delta v+\lambda v+\bar{\beta} \leq \bar{v}\left\{-c \frac{\dot{R}(t)}{R(t)}[M R(t) \operatorname{coth}(M R(t))-1]+\lambda-M^{2}\right\}
$$

Recalling that

$$
-\frac{\dot{R}(t)}{R(t)} \leq \tilde{\sigma}-\min \left(\bar{\sigma}, \sigma_{0}\right)=\tilde{\sigma}-\sigma_{0}
$$

and using the estimate

$$
0<\xi \operatorname{coth} \xi-1<\frac{1}{3} \xi^{2} \quad \forall \xi>0
$$

we get, for $c \leq \varepsilon$,

$$
c v_{t}-\Delta v+\lambda v+\bar{\beta} \leq \bar{v}\left\{\frac{1}{3} \varepsilon\left(\tilde{\sigma}-\sigma_{0}\right) M^{2} R^{2}(t)+\lambda-M^{2}\right\} .
$$

We shall now use the function $v$ to show that if $R_{1}$ is small enough then, for any $T_{1}>0$, the inequality

$$
R(t) \leq R_{1} \quad \text { cannot hold for all } t \geq T_{1}
$$

here $R_{1}$ is independent of the initial radius $R_{0}$.

Indeed, otherwise we deduce from (4.5), with $M^{2}=\lambda+1$, that

$$
c v_{t}-\Delta v+\lambda v+\bar{\beta} \leq 0 \quad \text { if } \quad 0 \leq r<R(t), \quad t>T_{1} .
$$


Since further $v=\bar{\sigma}$ on $r=R(t)$, the function

$$
v(r, t)-A e^{-\frac{\lambda}{c}\left(t-T_{1}\right)} \quad\left(A=\bar{\sigma}_{2}+\left|\sigma_{*}\right|\right)
$$

is a subsolution for $t \geq T_{1}$ and, by the maximum principle,

$$
\sigma(r, t) \geq v(r, t)-A e^{-\frac{\lambda}{c}\left(t-T_{1}\right)} .
$$

Using this in (1.16) we find (as in [9]) that $\dot{R}(t)>0$ if $t-T_{1}$ is sufficiently large, and this leads to a contradiction (as in [9]).

Having proved (4.6) we conclude that for any $T_{1}>0$ there exists a $T_{2}\left(>T_{1}\right)$ such that $R\left(T_{2}\right)>R_{1}$.

We choose positive constants $\delta_{1}$ and $M$ such that

$$
\delta_{1} \leq R_{1}
$$

and

$$
\frac{1}{3} \varepsilon\left(\tilde{\sigma}-\sigma_{0}\right) \delta_{1}^{2}<1, \quad M^{2}>\frac{\lambda}{1-\frac{1}{3} \varepsilon\left(\tilde{\sigma}-\sigma_{0}\right) \delta_{1}^{2}}
$$

Note that

$$
\xi \operatorname{coth} \xi=1+\frac{1}{3} \xi^{2}+O\left(\xi^{3}\right) \quad \text { if } \quad \xi \rightarrow 0
$$

so that, for some small positive constant $\delta_{2}$,

$$
\xi \operatorname{coth} \xi>1+\frac{1}{3}(1-\kappa) \xi^{2} \quad \text { if } \quad 0<\xi \leq \delta_{2}
$$

where

$$
\kappa=\frac{\bar{\sigma}-\tilde{\sigma}}{2 \bar{\sigma}_{2}+\left|\sigma_{*}\right|} ; \quad 0<\kappa<1 .
$$

We shall now prove the assertion (4.1) with

$$
\delta_{0}=\min \left\{\frac{\delta_{2}}{M}, \quad \delta_{1} \kappa^{\left(\tilde{\sigma}-\sigma_{0}\right) / \lambda}\right\}
$$

note that $\delta_{0}<\delta_{1} \leq R_{1}$.

Indeed, suppose (4.1) is not true. Then for any $T_{2}>0$ there exists $t_{0}>T_{2}$ such that $R\left(t_{0}\right)<\delta_{0}$. By the assertion we proved previously, we may choose $T_{2}$ such that $R\left(T_{2}\right)>R_{1}$. It then follows that there exists $t_{1} \in\left(T_{2}, t_{0}\right)$ such that $R\left(t_{1}\right)=\delta_{1}$ and $R(t) \leq \delta_{1}$ for all $t_{1} \leq t<t_{0}$. We claim that

$$
\sigma(r, t) \geq v(r, t)-A e^{-\frac{\lambda}{c}\left(t-t_{1}\right)} \quad \text { for } \quad 0 \leq r \leq R(t), \quad t_{1} \leq t \leq t_{0}
$$

where $v$ is the function defined in (4.2) and $A=\bar{\sigma}_{2}+\left|\sigma_{*}\right|$. Indeed, using (4.5) and the second inequality in (4.8) we see that the right-hand side of (4.12) is a subsolution to $\sigma$, so that (4.12) follows by comparison.

Now let $t_{2} \in\left(t_{1}, t_{0}\right)$ be such that $R\left(t_{2}\right)=\delta_{0}$ and $R(t)<\delta_{0}$ for all $t \in\left(t_{2}, t_{0}\right)$; then $\dot{R}\left(t_{2}\right) \leq 0$. Since $\dot{R}(t) / R(t) \geq-\left(\tilde{\sigma}-\sigma_{0}\right)$, we have

$$
R(t) \geq R\left(t_{1}\right) e^{-\left(\tilde{\sigma}-\sigma_{0}\right)\left(t-t_{1}\right)} \quad \text { if } \quad t \geq t_{1}
$$


and, consequently,

$$
t_{2}>t_{1}+\log \left(\frac{\delta_{1}}{\delta_{0}}\right)^{1 /\left(\tilde{\sigma}-\sigma_{0}\right)} \geq t_{1}+\log \kappa^{-1 / \lambda}
$$

by (4.11). Substituting (4.12) into (1.16) we get

$$
\begin{aligned}
\dot{R}(t) & =\frac{3}{(R(t))^{2}} \int_{0}^{R(t)}(\sigma(r, t)-\tilde{\sigma}) r^{2} d r \\
& \geq \frac{3 \bar{\sigma}_{2}}{R(t) \sinh (M R(t))} \int_{0}^{R(t)} r \sinh (M r) d r-\left(\tilde{\sigma}-\sigma_{*}\right) R(t)-A R(t) e^{-\lambda\left(t-t_{1}\right)} \\
& =\frac{3 \bar{\sigma}_{2}}{M^{2} R(t)}[M R(t) \operatorname{coth}(M R(t))-1]-\left(\tilde{\sigma}-\sigma_{*}\right) R(t)-A R(t) e^{-\lambda\left(t-t_{1}\right)}
\end{aligned}
$$

Since $R(t) \leq \delta_{0}$ for $t_{2} \leq t \leq t_{0}$, we have $M R(t) \leq M \delta_{0}<\delta_{2}$ by (4.11). Using also (4.9), (4.11) and (4.13), we conclude that

$$
\dot{R}(t)>(1-\kappa)\left(\bar{\sigma}-\sigma_{*}\right) R(t)-\left(\tilde{\sigma}-\sigma_{*}\right) R(t)-\kappa A R(t)
$$

if $t_{2} \leq t \leq t_{0}$. But since the right-hand side vanishes at $t=t_{2}$, by the choice $\kappa$ in (4.10), this is a contradiction to $\dot{R}_{2}\left(t_{0}\right) \leq 0$.

5. Asymptotic stability. In this section we shall extend the stability results of $\left(R_{1}\right)-\left(R_{4}\right)$ to the case where $c$ and $c^{\prime}$ are nonzero, but small. Thus we shall show that in cases $\left(\mathrm{R}_{1}\right),\left(\mathrm{R}_{2}\right)$, $\left(\mathrm{R}_{4}\right)$ (see Figures 1-4) the solutions $\left(\sigma_{s}, \beta_{s}, R_{s}\right)$ and, respectively, $\left(\sigma_{s}^{-}, \beta_{s}^{-}, R_{s}^{-}\right)$, are globally asymptotically stable and, respectively, locally asymptotically stable with domain of attraction $\left(0, R_{s}^{+}-\delta\right)$ for any $\delta>0$, provided $c$ and $c^{\prime}$ are positive and sufficiently small.

We first need to establish an upper bound on the solution, and this requires the following lemma.

LEMma 5.1. Let $(\sigma(r, t), \beta(r, t), R(t))$ be the solution of (1.12)-(1.18) for $0 \leq t<T_{0} \quad(0<$ $\left.T_{0} \leq \infty\right)$ and set

$$
\begin{aligned}
& w(r, t)=\frac{\bar{\beta} R(t)}{\sinh (\sqrt{\gamma} R(t))} \frac{\sinh (\sqrt{\gamma} r)}{r} \\
& v(r, t)=\left(1-\Lambda_{1}\right) \frac{\bar{\sigma} R(t)}{\sinh (\sqrt{\gamma} R(t))} \frac{\sinh (\sqrt{\gamma} r)}{r}+\Lambda_{1} \frac{\bar{\sigma} R(t)}{\sinh (\sqrt{\gamma} R(t))} \frac{\sinh (\sqrt{\gamma} r)}{r} .
\end{aligned}
$$

Assume that $|\dot{R}(t)| \leq L$ for $0 \leq t<T_{0}$ and

$$
\left|\varphi_{0}(r)-v(r, 0)\right| \leq M, \quad\left|\varphi_{0}(r)-w(r, 0)\right| \leq M \quad \text { for } \quad 0 \leq r \leq R_{0} .
$$

Then there exist positive constants $C$ (depending only on $\lambda, \gamma, \bar{\sigma}, \bar{\beta}$ ) and a (depending only on $\lambda, \gamma)$ such that

$$
\begin{aligned}
& |\beta(r, t)-w(r, t)| \leq C\left(L c^{\prime}+M e^{-\gamma t / c^{\prime}}\right), \\
& |\sigma(r, t)-v(r, t)| \leq C\left(L c^{\prime \prime}+M e^{-a t / c^{\prime \prime}}\right) \quad\left(c^{\prime \prime}=c+c^{\prime}\right)
\end{aligned}
$$

for $0 \leq r \leq R(t), 0 \leq t<T_{0}$. 
Proof. As easily verified,

$$
c^{\prime} w_{t}-\Delta w+\gamma w=-c^{\prime} \dot{R}(t) \cdot \gamma R(t) p(\sqrt{\gamma} R(t)) \cdot w
$$

where the function $p(\eta)$ is defined in (3.10). By Lemma 3.2

$$
0<\gamma R(t) p(\sqrt{\gamma} R(t)) \leq \sqrt{\gamma}
$$

and since $0<w \leq \bar{\beta}$, we get

$$
\left|c^{\prime} w_{t}-\Delta w+\gamma w\right| \leq L \bar{\beta} \sqrt{\gamma} c^{\prime}
$$

Consequently, by comparison,

$$
\pm(\beta(r, t)-w(r, t)) \leq \frac{L \bar{\beta} \sqrt{\gamma} c^{\prime}}{\gamma},
$$

which yields (again by comparison) the assertion (5.4).

Similarly,

$$
\begin{aligned}
c v_{t}-\Delta v+\lambda v+w= & -\left(1-\Lambda_{1}\right) c \dot{R} \cdot \lambda R p(\sqrt{\lambda} R) \cdot \frac{\bar{\sigma} R}{\sinh (\sqrt{\lambda} R)} \frac{\sinh (\sqrt{\lambda} r)}{r} \\
& -\Lambda_{1} c \dot{R} \cdot \gamma R p(\sqrt{\gamma} R) \frac{\bar{\sigma} R}{\sinh (\sqrt{\gamma} R)} \frac{\sinh (\sqrt{\gamma} r)}{r} \\
& +\Lambda_{1}(\lambda-\gamma) \bar{\sigma} \frac{R}{\sinh (\sqrt{\gamma} R)} \frac{\sinh (\sqrt{\gamma} r)}{r}+w,
\end{aligned}
$$

and since $\Lambda_{1}(\lambda-\gamma) \bar{\sigma}+\bar{\beta}=0$, we obtain

$$
\left|v_{t}-\Delta v+\lambda v+w\right| \leq L C|\bar{\sigma}| c \quad\left(C=\left(1+\left|\Lambda_{1}\right|\right) \sqrt{\lambda}+\left|\Lambda_{1}\right| \sqrt{\gamma}\right) .
$$

On the other hand, by (5.4),

$$
\left|c \sigma_{t}-\Delta \sigma+\lambda \sigma+w\right| \leq \frac{L \bar{\beta} c^{\prime}}{\sqrt{\gamma}}+M e^{-\gamma t / c^{\prime}}
$$

so that

$$
\left|c(\sigma-v)_{t}-\Delta(\sigma-v)+\lambda(\sigma-v)\right| \leq C L c^{\prime \prime}+M e^{-\gamma t / c^{\prime}} \leq C L c^{\prime \prime}+M e^{-\frac{\lambda \gamma t}{\lambda c^{\prime}+2 \gamma c}} .
$$

This implies that

$$
\pm(c V-\Delta V+\lambda V) \leq C L c^{\prime \prime} \quad \text { where } \quad V=\sigma-v-M_{1} e^{-\frac{\lambda \gamma t}{\lambda c^{\prime}+2 \gamma c}}
$$

and $M_{1}=M \max (1,2 / \lambda)$. By comparison (as in the derivation of (5.6)) we then get

$$
\pm V \leq \frac{C L c^{\prime \prime}}{\lambda}
$$

and (5.5) follows. 
We next establish, in all cases $\left(\mathrm{R}_{1}\right)-\left(\mathrm{R}_{4}\right)$, a uniform bound on $R(t)$ which is compatible with Figures 1-4:

THEOREM 5.2. Let $(\sigma(r, t), \beta(r, t), R(t))$ be the solution of (1.12)-(1.18) and let $R_{s}, R_{s}^{-}, R_{s}^{+}$ be as in Theorem 3.6. Let $K$ and $\delta$ be positive constants such that one of the following three conditions holds:

(i) $K \geq \delta+\max \left\{R_{0}, R_{s}\right\}$ if either $0 \leq(\phi-1) \Lambda_{1} \leq \phi, 0<\Lambda_{0}<\frac{1}{3}$ or $(\phi-1) \Lambda_{1}>\phi, 0 \leq \Lambda_{0}<\frac{1}{3}$;

(ii) $\max \left\{R_{0}, R_{s}^{-}\right\}+\delta \leq K \leq R_{s}^{+}-\delta$ if either $(\phi-1) \Lambda_{1}>\phi, \Lambda_{0}^{*}<\Lambda_{0}<0$ or $(\phi-1) \Lambda_{1}<-\frac{1}{\phi+1}$, $\frac{1}{3}<\Lambda_{0}<\Lambda_{0}^{* *}$

(iii) $R_{0}+\delta<K<R_{s}-\delta$ if either $-\frac{1}{\phi+1} \leq(\phi-1) \Lambda_{1}<0,0<\Lambda_{0}<\frac{1}{3}$ or $(\phi-1) \Lambda_{1}<-\frac{1}{\phi+1}$, $0<\Lambda_{0} \leq \frac{1}{3}$.

Then there exists a positive constant $\varepsilon_{0}$ depending only on $M$ (in (5.3)) and on $\lambda, \gamma, \bar{\sigma}, \bar{\beta}, \tilde{\sigma}, \delta, K$ such that if $c+c^{\prime} \leq \varepsilon_{0}$ then

$$
R(t) \leq K \quad \text { for all } \quad t \geq 0
$$

Proof. The assumptions on $\phi, \Lambda_{1}$ imply that

$$
A=\max \{\bar{\sigma}, 0\}-\tilde{\sigma}>0
$$

and that

$$
\text { either } \bar{\sigma}>0 \text { and } f(\sqrt{\lambda} K) \leq \Lambda_{0}-\tilde{\delta} \text {, or } \bar{\sigma}<0 \text { and } f(\sqrt{\lambda} K) \geq \Lambda_{0}+\tilde{\delta}
$$

where $\tilde{\delta}$ depends on the same constants on which $\varepsilon_{0}$ is asserted to depend on.

Suppose that (5.7) is not true. Then, since $K>R_{0}$, there exists a $t_{0}>0$ such that (5.7) holds for all $t<t_{0}$ and $R\left(t_{0}\right)=K$. Consequently,

$$
\dot{R}\left(t_{0}\right) \geq 0
$$

and, by Theorem 2.1,

$$
t_{0} \geq \frac{1}{A} \log \frac{K}{R_{0}}>\frac{1}{A} \log \frac{K}{K-\delta}=t_{*}>0 .
$$

By Theorem 2.1 we also have

$$
|\dot{R}(t)| \leq L \quad \text { if } \quad 0<t \leq t_{0}
$$

where

$$
L=\max \left\{|\bar{\sigma}-\tilde{\sigma}| K,\left|\tilde{\sigma}-\sigma_{0}\right| K\right\}
$$

It follows that $t_{*}$ and $L$ depend on the same constants upon which $\varepsilon_{0}$ is asserted to depend on.

We now apply Lemma 5.1 to obtain the inequalities

$$
v(r, t)-C\left(c^{\prime \prime}+e^{-a t / c^{\prime \prime}}\right) \leq \sigma(r, t) \leq v(r, t)+C\left(c^{\prime \prime}+e^{-a t / c^{\prime \prime}}\right)
$$

for $0 \leq r \leq R(t), 0<t \leq t_{0}$, where $v$ is the function defined in (5.2). Substituting the upper bound on $\sigma$ into (1.16) and setting $\eta(t)=\sqrt{\lambda} R(t)$, we get

$$
\frac{d \eta(t)}{d t} \leq \bar{\sigma} \eta(t)\left(f(\eta(t))-\Lambda_{0}\right)+C \eta(t)\left(c^{\prime \prime}+e^{-a t / c^{\prime \prime}}\right)
$$


Taking $t=t_{0}$ and using (5.8), (5.10) we get $\dot{\eta}\left(t_{0}\right)<0$ if $c^{\prime \prime} \leq \varepsilon_{0}$ and $\varepsilon_{0}$ is small enough, which is a contradiction to (5.9).

We now state the main result of this section which asserts, for $c, c^{\prime}$ small enough, the same stability results that hold in the case $c=c^{\prime}=0$ (see Figures 1-4).

Theorem 5.3. Let $(\sigma(r, t), \beta(r, t), R(t))$ and $R_{s}, R_{s}^{-}, R_{s}^{+}$be as in Theorem 5.2. Suppose the initial radius $R_{0}$ satisfies, for some small $\delta>0$, one of the following three conditions:

(i) $0<R_{0} \leq \frac{1}{\delta}$ if either $0 \leq(\phi-1) \Lambda_{1} \leq \phi, 0<\Lambda_{0}<\frac{1}{3}$ or $(\phi-1) \Lambda_{1}>\phi, 0 \leq \Lambda_{0}<\frac{1}{3}$;

(ii) $0<R_{0} \leq R_{s}^{+}-\delta$ if either $(\phi-1) \Lambda_{1}>\phi, \Lambda_{0}^{*}<\Lambda_{0}<0$ or $(\phi-1) \Lambda_{1}<-\frac{1}{\phi+1}, \frac{1}{3}<\Lambda_{0}<\Lambda_{0}^{* *}$;

(iii) $0<R_{0} \leq R_{s}-\delta$ if either $-\frac{1}{\phi+1} \leq(\phi-1) \Lambda_{1}<0,0<\Lambda_{0}<\frac{1}{3}$ or $(\phi-1) \Lambda_{1}<-\frac{1}{\phi+1}, 0<$ $\Lambda_{0} \leq \frac{1}{3}$.

Then there exists a positive constant $\varepsilon_{0}$ depending only on $M$ (in(5.3)), $\lambda, \gamma, \bar{\sigma}, \tilde{\sigma}, \bar{\beta}$ and $\delta$ such that if $c+c^{\prime} \leq \varepsilon_{0}$ then

$$
\lim _{t \rightarrow \infty} R(t)= \begin{cases}R_{s} & \text { in case (i) } \\ R_{s}^{-} & \text {in case (ii) } \\ 0 & \text { in case (iii) }\end{cases}
$$

moreover, the convergent is exponentially fast.

We shall first prove Theorem 5.3 in case (iii).

Proof of (5.15) in case (iii). Take a constant $K$ such that it satisfies the conditions in Theorem 5.2 (with $\delta$ replaced by $\frac{\delta}{2}$ ). By Theorem 5.2, $R(t) \leq K$ for all $t>0$ provided $c+c^{\prime} \leq \varepsilon_{0}, \varepsilon_{0}$ sufficiently small, and then (5.11)-(5.14) follow as before. Recall that in case (iii)

$$
\bar{\sigma} \leq \tilde{\sigma}<0 \quad \text { and } \quad f\left(\sqrt{\lambda} R_{0}\right)>\Lambda_{0} .
$$

Fix $t_{0}$ sufficiently small (independently of $\varepsilon_{0}$ ) so that, by Theorem 2.1,

$$
R(t) \leq R_{0} e^{-\tilde{\sigma} t}<K \quad \text { for } \quad 0 \leq t \leq t_{0} .
$$

Then

$$
f(\eta(t))-\Lambda_{0} \geq \beta_{0}>0 \quad\left(\beta_{0} \text { constant }\right)
$$

for all $0 \leq t \leq t_{0}$. On the other hand from (5.14) we get

$$
\dot{\eta}(t) \leq \bar{\sigma} \eta(t)\left[f(\eta(t))-\Lambda_{0}\right]+C \varepsilon_{0} \eta(t) \quad \text { if } \quad t \geq t_{0}
$$

Choosing $\varepsilon_{0}<|\bar{\sigma}| \beta_{0} /(2 C)$ we deduce that $\dot{\eta}(t)<0$ at $t=t_{0}$ and then, by continuity, $\dot{\eta}(t)<0$ in some interval $\left[t_{0}, t_{0}+\tau\right]$, so that $\eta(t) \leq \eta\left(t_{0}\right)<\sqrt{\lambda} K$ and

$$
\dot{\eta}(t)<\bar{\sigma} \eta(t)\left[f(\eta(t))-\Lambda_{0}\right]-\frac{1}{2} \bar{\sigma} \beta_{0} \eta(t)
$$

in this interval. We can now repeat the previous step and deduce, step-by-step, that $\dot{\eta}(t)<0$ and (5.17) holds for all $t \geq t_{0}$, so that $\eta(t) \rightarrow 0$ as $t \rightarrow \infty$ (and, in fact, exponentially fast since the right-hand side of (5.17) remains $\left.\leq-\frac{1}{2} \bar{\sigma} \beta_{0} \eta(t)\right)$.

REMARK 5.1. The above proof shows that $R(t)$ decreases monotonically (to zero) for all $t \geq 0$. 
We next consider case (i) of Theorem 5.3.

Lemma 5.4. Consider case (i) of Theorem 5.3. Then for an arbitrary $\alpha_{0}>0$ there exist positive constants $C, b$ and (sufficiently small) $\varepsilon_{0}$ depending only on $\lambda, \gamma, \bar{\sigma}, \tilde{\sigma}, \bar{\beta}, \delta$ and $\alpha_{0}$, such that the following is true if $c^{\prime \prime}=c+c^{\prime} \leq \varepsilon_{0}$ : For any $0<\alpha \leq \alpha_{0}$, if the inequalities

$$
\begin{aligned}
& \left|R(t)-R_{s}\right| \leq \alpha, \quad|\dot{R}(t)| \leq \alpha, \\
& \left|\sigma(r, t)-\sigma_{s}(r)\right| \leq \alpha, \quad\left|\beta(r, t)-\beta_{s}(r)\right| \leq \alpha
\end{aligned}
$$

hold for all $0 \leq r \leq R(t), t \geq 0$ then also the inequalities

$$
\begin{aligned}
& \left|R(t)-R_{s}\right| \leq C \alpha\left(c^{\prime \prime}+e^{-b t}\right), \quad|\dot{R}(t)| \leq C \alpha\left(c^{\prime \prime}+e^{-b t}\right), \\
& \left|\sigma(r, t)-\sigma_{s}(r)\right| \leq C \alpha\left(c^{\prime \prime}+e^{-b t}\right), \quad\left|\beta(r, t)-\beta_{s}(r)\right| \leq C \alpha\left(c^{\prime \prime}+e^{-b t}\right)
\end{aligned}
$$

hold for all $0 \leq r \leq R(t), t \geq 0$. Here $\sigma_{s}(r), \beta_{s}(r)$ are defined by (3.6), (3.7) for all $r>0$.

Proof. From Lemma 5.1 we get

$$
\begin{array}{ll}
|\sigma(r, t)-v(r, t)| \leq C \alpha\left(c^{\prime \prime}+e^{-a t / c^{\prime \prime}}\right) & \forall t \geq 0 \\
|\beta(r, t)-w(r, t)| \leq C \alpha\left(c^{\prime \prime}+e^{-\gamma t / c^{\prime \prime}}\right) & \forall t \geq 0 .
\end{array}
$$

Substituting (5.20) into (1.16) and setting $\eta(t)=\sqrt{\lambda} R(t)$, we get

$$
\left|\dot{\eta}(t)-\bar{\sigma} \eta(t)\left[f(\eta(t))-\Lambda_{0}\right]\right| \leq C \alpha \eta(t)\left(c^{\prime \prime}+e^{-a t / c^{\prime \prime}}\right) \quad \forall t \geq 0 .
$$

Consider first the case $0 \leq(\phi-1) \Lambda_{1} \leq \phi$. Then $\bar{\sigma}>0$ and

$$
f^{\prime}(\eta)<0 \quad \text { for all } \quad \eta>0 .
$$

By Theorems 4.1 and 5.2 we know that there are positive constants $\varepsilon_{0}, T_{0}, \delta_{0}$ and $K$ independent of $c, c^{\prime}$ and $\alpha$ (but dependent on $\alpha_{0}$ ) such that if $c^{\prime \prime} \leq \varepsilon_{0}$ then

$$
\delta_{0} \leq \eta(t) \leq K \quad \text { for all } \quad t \geq T_{0} .
$$

Consequently, by the mean value theorem,

$$
\bar{\sigma} \eta(t)\left[f(\eta(t))-\Lambda_{0}\right]= \begin{cases}\leq-C_{0}\left(\eta(t)-\eta_{s}\right), & \text { if } \quad \eta(t) \geq \eta_{s} \\ \geq-C_{0}\left(\eta(t)-\eta_{s}\right), & \text { if } \quad \eta(t)<\eta_{s}\end{cases}
$$

where $\eta_{s}=\sqrt{\lambda} R_{s}$ and $C_{0}$ is a positive constant depending only on $\bar{\sigma}, \delta_{0}, K$ and the coefficients of $f(\eta)$. We shall use this inequality to prove that there exist positive constants $\nu, B$ independent of $c, c^{\prime}$, and $\alpha$ such that

$$
\left|\eta(t)-\eta_{s}\right|<B \alpha\left(c^{\prime \prime}+e^{-\nu t}\right) \quad \text { for all } t \geq 0 .
$$

It is clear that (5.26) holds for all $0 \leq t \leq T_{0}$ if, for fixed $\nu>0, B$ is chosen sufficiently large. Therefore if (5.26) is not true then there exists a $t_{0}>T_{0}$ such that

$$
\eta_{s}-B \alpha\left(c^{\prime \prime}+e^{-\nu t}\right)<\eta(t)<\eta_{s}+B \alpha\left(c^{\prime \prime}+e^{-\nu t}\right) \quad \text { for } \quad 0 \leq t<t_{0}
$$


but not for $t=t_{0}$; for definiteness suppose that

$$
\eta\left(t_{0}\right)=\eta_{s}+B \alpha\left(c^{\prime \prime}+e^{-\nu t_{0}}\right)
$$

then also

$$
\dot{\eta}\left(t_{0}\right)+\nu B \alpha e^{-\nu t_{0}} \geq 0 .
$$

On the other hand, by (5.22) and (5.25),

$$
\dot{\eta}\left(t_{0}\right) \leq-C_{0}\left(\eta\left(t_{0}\right)-\eta_{s}\right)+C \alpha \eta\left(t_{0}\right)\left(c^{\prime \prime}+e^{-a t_{0} / \varepsilon_{0}}\right) .
$$

Substituting (5.27), (5.28) into this inequality and using the fact $\eta\left(t_{0}\right) \leq K$ we get

$$
-\nu B \alpha e^{-\nu t_{0}} \leq-C_{0} B \alpha\left(c^{\prime \prime}+e^{-\nu t_{0}}\right)+C K \alpha\left(c^{\prime \prime}+e^{-a t_{0} / \varepsilon_{0}}\right)
$$

which is a contradiction if we choose $\nu$ sufficiently small, say $\nu \leq \min \left(C_{0} / 2, a / \varepsilon_{0}\right)$, and $B$ correspondingly large. Having proved (5.26), the other estimates in (5.19) easily follow.

It remains to consider the case $(\phi-1) \Lambda_{1}>\phi$. Again we have $\bar{\sigma}>0$. But now (5.23) is not valid. However, if we denote by $\eta_{0}$ the stationary point of $f(\eta)$, then we still have

$$
f^{\prime}(\eta)<0 \quad \text { for all } \quad \eta \in\left(0, \eta_{0}\right),
$$

so that if $R_{0}<R_{*} \equiv \eta_{0} / \sqrt{\lambda}$ then the previous argument still works for the present situation. If on the other hand $R_{0} \geq R_{*}$, then $f(\eta(0))<0$ and thus $\eta(t)$ remains in the region where $f(\eta)$ is negative when $t$ varies in a small interval $\left[0, t_{0}\right]$. Using (5.22) for $t \geq t_{0}$ we conclude that if $c^{\prime \prime} \leq \varepsilon_{0}, \varepsilon_{0}$ is sufficiently small, then as long as $\eta(t) \geq \eta_{0}-2 \delta_{0}$ (for some small $\delta_{0}>0$ ) we have

$$
\dot{\eta}(t) \leq-\beta_{0} \eta(t)
$$

(for some positive constant $\left.\beta_{0}\right)$. Thus there exists a time $t_{*} \geq t_{0}$ such that $\eta(t)$ lies in $\left(0, \eta_{0}-\delta_{0}\right]$ at $t=t_{*}$. We can then proceed as in the former case to establish (5.26).

Having proved Lemma 5.4, we can now apply it successively as in [9] (over intervals $\left[t_{n}, \infty\right.$ ) with increasing $n$ ) and establish (5.15) in case (i).

We shall next extend Lemma 5.4 to case (ii).

Lemma 5.5. Consider case (ii) of Theorem 5.3. Then for an arbitrary $\alpha_{0}$ there exist positive constants $C, b$ and (sufficiently small) $\varepsilon_{0}$ depending on $\lambda, \gamma, \bar{\sigma}, \tilde{\sigma}, \bar{\beta}, \delta$ and $\alpha_{0}$ such that the following is true if $c^{\prime \prime}=c+c^{\prime} \leq \varepsilon_{0}$ : For any $0<\alpha<\alpha_{0}$, if the inequalities

$$
\begin{aligned}
& \left|R(t)-R_{s}^{-}\right| \leq \alpha, \quad|\dot{R}(t)| \leq \alpha, \\
& \left|\sigma(r, t)-\sigma_{s}^{-}(r)\right| \leq \alpha, \quad\left|\beta(r, t)-\beta_{s}^{-}(r)\right| \leq \alpha
\end{aligned}
$$

hold for all $0 \leq r \leq R(t), t \geq 0$ then also the inequalities

$$
\begin{aligned}
& \left|R(t)-R_{s}^{-}\right| \leq C \alpha\left(c^{\prime \prime}+e^{-b t}\right), \quad|\dot{R}(t)| \leq C \alpha\left(c^{\prime \prime}+e^{-b t}\right) \\
& \left|\sigma(r, t)-\sigma_{s}^{-}(r)\right| \leq C \alpha\left(c^{\prime \prime}+e^{-b t}\right), \quad\left|\beta(r, t)-\beta_{s}^{-}(r)\right| \leq C \alpha\left(c^{\prime \prime}+e^{-b t}\right)
\end{aligned}
$$

hold for all $0 \leq r \leq R(t), t \geq 0$.

Proof. The proof is similar to the proof of Lemma 5.4 for the case $(\phi-1) \Lambda_{1}>\phi$.

Again, by using Lemma 5.5 and following a similar argument as in [9] we can derive the assertion (5.15) in case (ii). 
6. Instability: Unboundedness of $\mathbf{R}(\mathbf{t})$. We shall use the notation

$$
w_{\mu}(r, t)=\frac{R(t)}{\sinh (\sqrt{\mu} R(t))} \frac{\sinh (\sqrt{\mu} r)}{r} \quad \text { for any } \mu>0,
$$

where $(\sigma(r, t), \beta(r, t), R(t))$ is the solution of (1.12)-(1.18). We set

$$
\begin{aligned}
& w(r, t)=\bar{\beta} w_{\gamma}(r, t), \\
& v(r, t)=\bar{\sigma}\left\{\left(1-\Lambda_{1}\right) w_{\lambda}(r, t)+\Lambda_{1} w_{\gamma}(r, t)\right\}
\end{aligned}
$$

and

$$
w_{0}(r)=w(r, 0), \quad v_{0}(r)=v(r, 0) .
$$

We shall denote the set of parameters $\lambda, \gamma, \bar{\sigma}, \tilde{\sigma}, \bar{\beta}, R_{0}$ by $\mathcal{A}$, i.e., $\mathcal{A}=\left\{\lambda, \gamma, \bar{\sigma}, \tilde{\sigma}, \bar{\beta}, R_{0}\right\}$.

In this section we consider essentially all the cases that were not covered by the stability results of $\S 5$. We can divide them into four disjoint cases:

(i) $0 \leq(\phi-1) \Lambda_{1} \leq \phi, \Lambda_{0}<0,0<R_{0}<\infty$;

(ii) $(\phi-1) \Lambda_{1}>\phi$ and either $\Lambda_{0}^{*}<\Lambda_{0}<0, R_{0}>R_{s}^{+}$or $\Lambda_{0}<\Lambda_{0}^{*}, \quad 0<R_{0}<\infty$;

(iii) $-\frac{1}{\phi+1} \leq(\phi-1) \Lambda_{1}<0$ and either $\Lambda_{0} \geq \frac{1}{3}, 0<R_{0}<\infty$, or $0<\Lambda<\frac{1}{3}, R_{0}>R_{s}$, and

(iv) $(\phi-1) \Lambda_{1}<-\frac{1}{\phi+1}$ and $0<\Lambda_{0} \leq \frac{1}{3}, R_{0}>R_{s}$, or $\frac{1}{3}<\Lambda_{0}<\Lambda_{0}^{* *}, R_{0}>R_{s}^{+}$, or $\Lambda_{0}>\Lambda_{0}^{* *}$, $0<R_{0}<\infty$.

We want to show that if one of these conditions is satisfied then there are initial data $\varphi_{0}(r), \psi_{0}(r)$ for which $R(t) \rightarrow \infty$ as $t \rightarrow \infty$.

We take $\varphi_{0}, \psi_{0}$ such that

$$
\begin{aligned}
& \varphi_{0}(r)-\frac{1}{\gamma-\lambda} \psi_{0}(r) \geq\left(\bar{\sigma}-\frac{\bar{\beta}}{\gamma-\lambda}\right) \frac{R_{0} \sinh (\sqrt{\lambda} r)}{r \sinh \left(\sqrt{\lambda} R_{0}\right)}, \\
& \psi_{0}(r)=\left\{\begin{array}{ccc}
\geq \frac{\bar{\beta} R_{0} \sinh (\sqrt{\gamma} r)}{r \sinh \left(\sqrt{\gamma} R_{0}\right)} & \text { if } & \gamma>\lambda \\
\leq \frac{\bar{\beta} R_{0} \sinh (\sqrt{\gamma} r)}{r \sinh \left(\sqrt{\gamma} R_{0}\right)} & \text { if } & \gamma<\lambda
\end{array}\right.
\end{aligned}
$$

and

$$
\Delta \psi_{0}(r)-\gamma \psi_{0}(r) \leq 0
$$

for $0 \leq r \leq R_{0}$.

THEOREM 6.1. Assume that one of the conditions (i),...,(iv) is satisfied and that (6.5)(6.7) hold. If $0<c^{\prime} \leq c \leq \varepsilon_{0}$ when $\varepsilon_{0}$ is sufficiently small depending on the parameter set $\mathcal{A}$, then

$$
\begin{aligned}
& \dot{R(t)}>0 \quad \text { for all } t>0, \\
& R(t) \rightarrow \infty \quad \text { if } t \rightarrow \infty .
\end{aligned}
$$

REmark 6.1. The condition $c^{\prime} \leq c$ is a technical limitation of the proof. It means that the inhibitor diffuses faster than the nutrient. 
REMARK 6.2. From the proof of the theorem it follows that if one of the conditions (i)-(iv) is satisfied with $R_{0}-\delta$ instead of $R_{0}$, for some $\delta>0$, then $\varepsilon_{0}$ depends on $\delta$ but not on the specific $R_{0}$.

We shall need the following lemma:

LEMma 6.2. Under assumptions of Theorem 6.1, for any given $M_{1}>R_{0}$ there exist $\varepsilon_{0}>0$ and $T_{0}>0$ such that

$$
\dot{R}(t)>0 \quad \text { if } \quad 0 \leq t \leq T_{0} \quad \text { and } \quad R\left(T_{0}\right) \geq M_{1},
$$

provided $c+c^{\prime} \leq \varepsilon_{0}$.

Proof. From (6.5), (6.6) it follows that

$$
\varphi_{0}(r) \geq\left(1-\Lambda_{1}\right) \frac{\bar{\sigma} R_{0} \sinh (\sqrt{\lambda} r)}{r \sinh (\sqrt{\lambda} r)}+\Lambda_{1} \frac{\bar{\sigma} R_{0} \sinh (\sqrt{\gamma} r)}{r \sinh \left(\sqrt{\gamma} R_{0}\right)} .
$$

Substituting this into (1.16) we get

$$
\dot{R}(0) \geq \bar{\sigma} R_{0}\left[f\left(\sqrt{\lambda} R_{0}\right)-\Lambda_{0}\right]>0 .
$$

Hence there exists a $t_{0}>0$ such that $\dot{R}(t)>0$ for $0 \leq t \leq t_{0}$. Now, for a given $M_{1}>R_{0}$, let $T_{0}=\frac{1}{\mu_{0}} \log \frac{M_{1}}{R_{0}}+t_{0}$, where

$$
\mu_{0}=\frac{1}{2} \bar{\sigma}\left[f\left(\sqrt{\lambda} R_{0}\right)-\Lambda_{0}\right]>0 .
$$

From Theorem 2.1 we see that for all $0 \leq t \leq T_{0}$,

$$
R(t) \leq R_{0} e^{A T_{0}} \quad(A=\max (\bar{\sigma}, 0)-\tilde{\sigma}>0)
$$

which implies

$$
|\dot{R}(t)| \leq A_{1} R_{0} e^{A T_{0}} \quad \text { for all } \quad 0 \leq t \leq T_{0}
$$

where $A_{1}$ is a constant depending only on $\bar{\sigma}, \tilde{\sigma}$ and $\sigma_{0}$. Therefore, applying Lemma 5.1 (taking $M=\bar{\beta}$ ) we get

$$
\sigma(r, t) \geq v(r, t)-C c^{\prime \prime} \quad \text { for all } t_{0} \leq t \leq T_{0}
$$

where $c^{\prime \prime}=c+c^{\prime}$ and $C$ is a constant depending only on $\mathcal{A}, t_{0}$ and $T_{0}$. Substituting this estimate into (1.16) we find, as before, that

$$
\dot{R}(t) \geq R(t)\left\{\bar{\sigma}\left[f(\sqrt{\lambda} R(t))-\Lambda_{0}\right]-C c^{\prime \prime}\right\} \quad \text { for all } \quad t_{0} \leq t \leq T_{0} .
$$

From this inequality, it follows that, provided $c^{\prime \prime} \leq \varepsilon_{0} \equiv \mu_{0} / C$, as long as $\dot{R}(t)$ remains positive for $t \geq t_{0}$ and $\leq T_{0}$ we have $R(t)>R_{0}$, so that

$$
\bar{\sigma}\left(f(\sqrt{\lambda} R(t))-\Lambda_{0}\right) \geq 2 \mu_{0}
$$

and thus $\dot{R}(t) \geq \mu_{0} R(t)>0$. Finally, integrating the last inequality in the interval $t_{0} \leq t \leq T_{0}$ and using the definition of $T_{0}$ we obtain the assertion $R\left(T_{0}\right) \geq M_{1}$.

Proof of Theorem 6.1. We shall specify $M_{1}$ later on, and then $\varepsilon_{0}$ and $T_{0}$ will be chosen, accordingly, as in Lemma 6.2. Let $t_{*}$ be any number larger than $T_{0}$ such that

$$
\dot{R}(t)>0 \quad \text { if } \quad 0 \leq t<t_{*} .
$$


If we prove that $\dot{R}\left(t_{*}\right)>0$, then a continuity argument shows that (6.8) is satisfied. Then also (6.9) holds since, otherwise, $R^{*}=\lim _{t \rightarrow \infty} R(t)<\infty$ and the corresponding limits of $\sigma(r, t), \beta(r, t)$ as $t \rightarrow \infty$ (which exist by standard parabolic theory [10]) form a stationary solution, which is a contradiction.

Notice that (6.10) implies that

$$
R_{0} \leq R(t) \leq R\left(t_{*}\right) \equiv M \quad\left(0 \leq t \leq t_{*}\right) \quad \text { and } \quad M \geq M_{1} .
$$

To prove that $\dot{R}\left(t_{*}\right)>0$ we consider first the case $\bar{\sigma}>0$ (Figures 1,2 ) and divide it into three cases. The first one is

(a) : $\quad \gamma>\lambda, \quad \bar{\sigma} \geq \frac{\bar{\beta}}{\gamma-\lambda}$,

i.e., $\phi>1,0 \leq \Lambda_{1} \leq 1$ (For this case Lemma 6.2 is not needed). Since $\dot{R}(t) \geq 0$ for $0 \leq t \leq t_{*}$,

$$
c^{\prime} w_{t}-\Delta w+\gamma w \leq 0 \quad \text { if } \quad 0 \leq r \leq R(t), 0 \leq t \leq t_{*} .
$$

We claim that

$$
\beta_{t}(r, t) \leq 0 \quad \text { if } \quad 0 \leq r \leq R(t), 0 \leq t \leq t_{*} .
$$

Indeed, the function $u=\beta_{t}$ satisfies

$$
c^{\prime} u_{t}=\Delta u-\gamma u
$$

and, by differentiating (1.14),

$$
\frac{\partial u}{\partial r}(0, t)=0, \quad u(R(t), t)=-\beta_{r}(R(t), t) \dot{R}(t) \leq 0
$$

since $\dot{R}(t) \geq 0$ and $\beta_{r}(R(t), t)>0$ by the maximum principle. Finally (6.7) ensures that $u(r, 0) \leq 0$ and, then, (6.13) follows by the maximum principle applied to $u$.

From (1.13), (6.13) we deduce that

$$
c \beta_{t}-\Delta \beta+\gamma \beta=\left(c-c^{\prime}\right) \beta_{t} \leq 0
$$

so that, by comparison,

$$
\beta(r, t) \geq w(r, t)
$$

Consider next the function

$$
z(r, t)=\sigma(r, t)-\frac{1}{\gamma-\lambda} \beta(r, t) .
$$

By (1.12), (6.14) we have

$$
c z_{t}-\Delta z+\lambda z \geq 0
$$

and, since $\bar{\sigma}-\bar{\beta} /(\gamma-\lambda) \geq 0$,

$$
z(r, t) \geq\left(\bar{\sigma}-\frac{\bar{\beta}}{\gamma-\lambda}\right) w_{\lambda}(r, t)
$$

by comparison. Combining this with (6.15) we conclude that

$$
\sigma(r, t)=z(r, t)+\frac{1}{\gamma-\lambda} \beta(r, t) \geq v(r, t) .
$$


Substituting this estimate into (1.16) we obtain the inequality

$$
\dot{R}(t) \geq \bar{\sigma} R(t)\left[f(\sqrt{\lambda} R(t))-\Lambda_{0}\right] \quad \text { for } \quad 0 \leq t \leq t_{*},
$$

and, since

$$
f(\sqrt{\lambda} R(t))-\Lambda_{0}>0
$$

it follows that $\dot{R}\left(t_{*}\right)>0$.

In the sequel we shall use the fact that

$$
\frac{R \sinh (\sqrt{\mu} r)}{r \sinh (\sqrt{\mu} R)} \geq \frac{R \sinh (\sqrt{\lambda} r)}{r \sinh (\sqrt{\lambda} R)} \quad \text { if } \quad \mu<\lambda, 0 \leq r \leq R .
$$

The proof is by comparison: If we denote the right-hand side by $u$ and the left-hand side by $v$, then

$$
\Delta v-\mu v=0, \quad \Delta u-\mu u=(\lambda-\mu) u \geq 0 \quad \text { if } \quad 0 \leq r \leq R
$$

since further $u=v$ on $r=R$, the assertion (6.17) follows.

Consider next the case:

(b) : $\quad \gamma>\lambda, \quad \bar{\sigma}<\frac{\bar{\beta}}{\gamma-\lambda}$,

i.e. $\phi>1, \Lambda_{1}>1$.

By Theorem 2.1

$$
\dot{R}(t) \leq A R(t) \quad \text { for all } t>0,
$$

where $A=\bar{\sigma}-\tilde{\sigma}>0$. Since $\lim _{\eta \rightarrow \infty} p(\eta)=0$ and $\Lambda_{0}<0$, we can choose $M_{1}$ sufficiently large and $\varepsilon_{0}$ small enough so that

$$
\left(1-\Lambda_{1}\right) p\left(\frac{\lambda M_{1}}{\varepsilon_{0} A M_{1}+\sqrt{\lambda}}\right)>\Lambda_{0}
$$

( $\varepsilon_{0}$ has also to be small enough as required by Lemma 6.2.) Recalling that $\Lambda_{1}$ is positive, $p$ is monotone decreasing and that $M \geq M_{1}$, we then also have

$$
\left(1-\Lambda_{1}\right) p\left(\frac{\lambda M}{\varepsilon_{0} A M+\sqrt{\lambda}}\right)+\Lambda_{1} p(\sqrt{\lambda} M)>\Lambda_{0} .
$$

Let

$$
\lambda_{1}=\left(\frac{\lambda}{\varepsilon_{0} A M+\sqrt{\lambda}}\right)^{2} .
$$

Clearly $0<\lambda_{1}<\lambda$ and, since $\lambda=\varepsilon_{0} \sqrt{\lambda_{1}} A M+\sqrt{\lambda_{1}} \sqrt{\lambda}$,

$$
\lambda-\lambda_{1}-\varepsilon_{0} \sqrt{\lambda_{1}} A M \geq 0 \text {. }
$$

Introduce the function

$$
Z=\left(\bar{\sigma}-\frac{\bar{\beta}}{\gamma-\lambda}\right) w_{\lambda_{1}}(r, t)
$$

It satisfies

$$
\begin{aligned}
c Z_{t}-\Delta Z+\lambda Z & =\left[-c \sqrt{\lambda_{1}} \dot{R}(t) \frac{\sqrt{\lambda_{1}} R(t) \operatorname{coth}\left(\sqrt{\lambda_{1}} R(t)\right)-1}{\sqrt{\lambda_{1}} R(t)}-\lambda_{1}+\lambda\right]\left(\bar{\sigma}-\frac{\bar{\beta}}{\gamma-\lambda}\right) w_{\lambda_{1}} \\
& \leq\left[-\varepsilon_{0} \sqrt{\lambda_{1}} A M-\lambda_{1}+\lambda\right]\left(\bar{\sigma}-\frac{\bar{\beta}}{\gamma-\lambda}\right) w_{\lambda_{1}} \leq 0
\end{aligned}
$$


by condition (b), (6.18), (6.21) and the inequality $\xi \operatorname{coth} \xi-1 \leq \xi$. Also

$$
\begin{aligned}
Z(r, 0) & =\left(\bar{\sigma}-\frac{\bar{\beta}}{\gamma-\lambda}\right) \frac{R_{0} \sinh \left(\sqrt{\lambda_{1}} r\right)}{r \sinh \left(\sqrt{\lambda_{1}} R_{0}\right)} \leq\left(\bar{\sigma}-\frac{\bar{\beta}}{\gamma-\lambda}\right) \frac{R_{0} \sinh (\sqrt{\lambda} r)}{r \sinh \left(\sqrt{\lambda} R_{0}\right)} \\
& \leq \varphi_{0}(r)-\frac{1}{\gamma-\lambda} \psi_{0}(r)=z(r, 0),
\end{aligned}
$$

and $Z(R(t), t)=z(R(t), t)$ when $z$ is the function defined in (6.16). Hence, by comparison, $z(r, t) \geq Z(r, t)$, so that

$$
\begin{aligned}
\sigma(r, t) & =z(r, t)+\frac{1}{\gamma-\lambda} \beta(r, t) \\
& \geq\left(\bar{\sigma}-\frac{\bar{\beta}}{\gamma-\lambda}\right) \frac{R(t) \sinh \left(\sqrt{\lambda_{1}} r\right)}{r \sinh \left(\sqrt{\lambda_{1}} R(t)\right)}+\left(\frac{\bar{\beta}}{\gamma-\lambda}\right) \frac{R(t) \sinh (\sqrt{\gamma} r)}{r \sinh (\sqrt{\gamma} R(t))} .
\end{aligned}
$$

Substituting this estimate into (1.16) and using (6.19) we obtain, at $t=t_{*}$,

$$
\begin{aligned}
\dot{R}\left(t_{*}\right) & =\frac{3}{M^{2}} \int_{0}^{M}\left(\sigma\left(r, t_{*}\right)-\tilde{\sigma}\right) r^{2} d r \\
& \geq \bar{\sigma} M\left[\left(1-\Lambda_{1}\right) p\left(\sqrt{\lambda_{1}} M\right)+\Lambda_{1} p(\sqrt{\gamma} M)-\Lambda_{0}\right] \\
& =\bar{\sigma} M\left[\left(1-\Lambda_{1}\right) p\left(\frac{\lambda M}{\varepsilon_{0} A M+\sqrt{\lambda}}\right)+\Lambda_{1} p(\sqrt{\gamma} M)-\Lambda_{0}\right]>0 .
\end{aligned}
$$

The third case is

(c) : $\quad \gamma<\lambda$,

i.e., $\phi<1, \Lambda_{1}<0$. We now take $M_{1}$ sufficiently large and $\varepsilon_{0}$ sufficiently small so that

$$
\Lambda_{1} p\left(\frac{\gamma M_{1}}{\varepsilon_{0} A M_{1}+\sqrt{\gamma}}\right)>\Lambda_{0},
$$

and, since $1-\Lambda_{1}>0$ and $M \geq M_{1}$, we have

$$
\left(1-\Lambda_{1}\right) p(\sqrt{\lambda} M)+\Lambda_{1} p\left(\frac{\gamma M}{\varepsilon_{0} A M+\sqrt{\gamma}}\right)>\Lambda_{0} ;
$$

$\varepsilon_{0}$ is also chosen small enough as required in Lemma 6.2.

Let

$$
\gamma_{1}=\left(\frac{\gamma}{\varepsilon_{0} A M+\sqrt{\gamma}}\right)^{2}
$$

so that, as before,

$$
\gamma-\gamma_{1}-\varepsilon_{0} \sqrt{\gamma_{1}} A M \geq 0 .
$$

Setting

$$
B(r, t)=\bar{\beta} w_{\gamma_{1}}(r, t)
$$

and proceeding as in the case of $Z$ above, we deduce, using (6.24), that

$$
c B_{t}-\Delta B+\gamma B \geq\left[-\varepsilon_{0} \sqrt{\gamma_{1}} A M-\gamma_{1}+\gamma\right] \bar{\beta} w_{\gamma_{1}} \geq 0 .
$$


Since also

$$
B(r, 0)=\frac{\bar{\beta} R_{0} \sinh \left(\sqrt{\gamma_{1}} r\right)}{r \sinh \left(\sqrt{\gamma_{1}} R_{0}\right)} \geq \frac{\bar{\beta} R_{0} \sinh (\sqrt{\gamma} r)}{r \sinh \left(\sqrt{\gamma} R_{0}\right)} \geq \psi_{0}(r),
$$

we get, by comparison,

$$
\beta(r, t) \leq B(r, t)
$$

and, consequently,

$$
\begin{aligned}
\sigma(r, t) & =z(r, t)-\frac{1}{\lambda-\gamma} \beta(r, t) \\
& \geq\left(\bar{\sigma}-\frac{\bar{\beta}}{\gamma-\lambda}\right) \frac{R(t) \sinh (\sqrt{\lambda} r)}{r \sinh (\sqrt{\lambda} R(t))}-\frac{\bar{\beta}}{\lambda-\gamma} \frac{R(t) \sinh \left(\sqrt{\gamma_{1}} r\right)}{r \sinh \left(\sqrt{\gamma_{1}} R(t)\right)}
\end{aligned}
$$

for $0 \leq r \leq R(t), 0 \leq t \leq t_{*}$. Substituting this into (1.16) at $t=t_{*}$ and using (6.23), we find that $\dot{\dot{R}}\left(t_{*}\right)>0$.

So far we have dealt with the case $\bar{\sigma}>0$. Consider next the case where $\bar{\sigma}<0$. We again divide it into three cases:

$$
\begin{array}{ll}
\left(\mathrm{a}^{\prime}\right): & \gamma<\lambda, \quad \bar{\sigma} \leq \frac{\bar{\beta}}{\gamma-\lambda} \quad\left(\Longleftrightarrow \phi<1,0<\Lambda_{1} \leq 1\right), \\
\left(\mathrm{b}^{\prime}\right): & \gamma<\lambda, \quad \bar{\sigma}>\frac{\bar{\beta}}{\gamma-\lambda} \quad\left(\Longleftrightarrow \phi<1, \Lambda_{1}>1\right), \\
\left(\mathrm{c}^{\prime}\right): & \gamma>\lambda, \quad\left(\Longleftrightarrow \Lambda_{1}<0\right) .
\end{array}
$$

In case $\left(\mathrm{a}^{\prime}\right)$ we choose $M_{1}$ sufficiently large and $\varepsilon_{0}$ small so that, for $M \geq M_{1}$,

$$
\left(1-\Lambda_{1}\right) p\left(\frac{\lambda M}{\varepsilon_{0} A M+\sqrt{\lambda}}\right)+\Lambda_{1} p\left(\frac{\gamma M}{\varepsilon_{0} A M+\sqrt{\gamma}}\right)<\Lambda_{0} \quad(A=-\tilde{\sigma}>0) ;
$$

then, in particular, (6.27) holds for $M=R\left(t_{*}\right)$ as in (6.11). We now proceed to estimate $z$ from below by $Z$ and $\beta$ from above by $B$, as before. This enables us to derive a lower bound on $\sigma$, as before, which leads to $\dot{R}\left(t_{*}\right)>0$.

In case $\left(\mathrm{b}^{\prime}\right)$ we choose $M_{1}$ and $\varepsilon_{0}$ such that

$$
\Lambda_{1} p\left(\frac{\gamma M_{1}}{\varepsilon_{0} A M_{1}+\sqrt{\gamma}}\right)<\Lambda_{0}
$$

since $1-\Lambda_{1}<0$ and $M \geq M_{1}$, we then also have

$$
\left(1-\Lambda_{1}\right) p(\sqrt{\lambda} M)+\Lambda_{1} p\left(\frac{\gamma M}{\varepsilon_{0} A M+\sqrt{\gamma}}\right)<\Lambda_{0} .
$$

Next we estimate $\beta$ from above by $B$ and $z /(\bar{\sigma}-\bar{\beta} /(\gamma-\lambda))$ from above by $w_{\lambda}$ as before, and using the inequalities in ( $\left.\mathrm{b}^{\prime}\right)$ obtain a lower bound on $\sigma$. Substituting this into (1.16), we derive, with the aid of (6.28), the inequality $\dot{R}\left(t_{*}\right)>0$.

Finally, in case $\left(\mathrm{c}^{\prime}\right)$ we choose $M_{1}$ and $\varepsilon_{0}$ such that

$$
\left(1-\Lambda_{1}\right) p\left(\frac{\lambda M_{1}}{\varepsilon_{0} A M_{1}+\sqrt{\lambda}}\right)<\Lambda_{0}
$$


so that, since $\Lambda_{1}<0$ and $M \geq M_{1}$,

$$
\left(1-\Lambda_{1}\right) p\left(\frac{\lambda M}{\varepsilon_{0} A M+\sqrt{\lambda}}\right)+\Lambda_{1} p(\sqrt{\gamma} M)<\Lambda_{0} .
$$

Next we proceed to estimate $z$ from below by $Z$ and use (6.15), in order to derive a lower bound on $\sigma$, which leads to $\dot{R}\left(t_{*}\right)>0$ (by $(1.16),(6.29)$ ).

We finally note that the case $\bar{\sigma}=0$ can be handled similarly to the case $\bar{\sigma}>0$, or $\bar{\sigma}<0$. $\square$

REMARK 6.3. Throughout this paper we assume that $\gamma \neq \lambda$. For completeness we mention that the case $\gamma=\lambda$, or $\phi=1$, can be handled by letting $\phi \rightarrow 1$. By L'Hospital's rule we find that

$$
f(\eta)=p(\eta)+\frac{\bar{\beta}}{\lambda \bar{\sigma}} \frac{\eta p^{\prime}(\eta)}{2}
$$

and the stability and instability of stationary solutions follows by the same analysis as above applied to this limit function $f$.

7. The effect of inhibitor's parameters on tumor's growth. We may view the parameter $\Lambda_{0}$ as the tumor's characteristic (without inhibitors), the parameter $\gamma$ as the inhibitor's (drug) characteristic, and the parameter $\bar{\beta}$ as the external inhibitor (normalized) concentration. In this section, building upon the mathematical results of $\S \S 3-6$, we shall determine how the tumor's growth depends upon the parameters $\bar{\beta}$ and $\gamma($ or $\phi)$; this will suggest a strategy for drug treatment.

Note that we are concerned here with large time progress of the therapy and therefore the parameters $c$ and $c^{\prime}$ will not play a role in the discussion of the drug treatment; we assume that they are small enough as in sections 5,6 .

By (1.19) we can write

$$
\Lambda_{1}=\frac{1}{\lambda \bar{\sigma}} \frac{\bar{\beta}}{\phi^{2}-1}, \quad \phi=\sqrt{\frac{\gamma}{\lambda}} .
$$

We shall first consider the case

$$
\bar{\sigma}>0
$$

we are then in the situations described in Figures 1 and 2.

Assumption (7.2) implies that

$$
\begin{cases}\Lambda_{1}>0 & \text { if and only if } \quad \phi>1 \\ \Lambda_{1}<0 & \text { if and only if } \quad \phi<1\end{cases}
$$

a property that we shall often be using in the sequel. According to $\S 3$, stationary solutions exist in just two cases:

$\underline{\text { Case A }} \quad 0<\Lambda_{0}<\frac{1}{3} \quad$ (Figures 1 and $\left.2(\mathrm{a})\right)$,

Case B $\quad \Lambda_{0}^{*}<\Lambda_{0}<0 \quad$ (Figure 2(b))

(we omit for simplicity the cases $\Lambda_{0}=0, \Lambda_{0}=\Lambda_{0}^{*}$ ).

In case A there exists a unique stationary solution, with normalized radius $\eta=\sqrt{\lambda} R_{s}$, and it is globally asymptotically stable, whereas in case B there are two stationary solutions with normalized radii

$$
\eta^{-}=\sqrt{\lambda} R_{s}^{-}, \quad \eta^{+}=\sqrt{\lambda} R_{s}^{+} \quad\left(R_{s}^{-}<R_{s}^{+}\right)
$$


the one with smaller radius is asymptotically stable if $R_{0}<R_{s}^{-}$and the one with larger radius is unstable and, in fact, for the time-dependent solution, if $R_{0}>R_{s}^{+}$then $R(t)$ may grow to $\infty$ as $t \rightarrow \infty$.

Taking (7.1) into consideration, we can rewrite the function $f(\eta)$ defined by $(3.11),(3.10)$ as follows:

$$
f(\eta)=p(\eta)+\frac{\bar{\beta}}{\lambda \bar{\sigma}} \frac{p(\phi \eta)-p(\eta)}{\phi^{2}-1}, \quad \phi=\sqrt{\frac{\gamma}{\lambda}} .
$$

We shall write $f(\eta)$ as $f(\eta, \bar{\beta}, \phi)$ to emphasize the dependence on the relevant parameters regarded as independent variables. We shall indicate the dependency of $\eta$ and $\eta^{ \pm}$on $\bar{\beta}, \phi, \gamma$ by writing

$$
\left\{\begin{array}{l}
\eta=\eta(\bar{\beta}, \phi)=\eta[\bar{\beta}, \gamma] \\
\eta^{ \pm}=\eta^{ \pm}(\bar{\beta}, \phi)=\eta^{ \pm}[\bar{\beta}, \gamma] .
\end{array}\right.
$$

At the point $\eta_{0}$ where $f$ takes its minimum (Theorem 3.5(ii)) we have

$$
\left.\frac{\partial f}{\partial \eta}\right|_{\eta=\eta_{0}}=p^{\prime}\left(\eta_{0}\right)+\frac{\bar{\beta}}{\lambda \bar{\sigma}} \frac{\phi p^{\prime}\left(\phi \eta_{0}\right)-p^{\prime}\left(\eta_{0}\right)}{\phi^{2}-1}=0 .
$$

We shall write $\eta_{0}=\eta_{0}(\bar{\beta}, \phi)$ and denote the minimum of $f$ by $\Lambda_{0}^{*}=\Lambda_{0}^{*}(\bar{\beta}, \phi)=\Lambda_{0}^{*}[\bar{\beta}, \gamma]$, so that

$$
\Lambda_{0}^{*}=\Lambda_{0}^{*}(\bar{\beta}, \phi)=f\left(\eta_{0}(\bar{\beta}, \phi), \bar{\beta}, \phi\right) .
$$

The following lemma will play an important role in our discussion.

LEMma 7.1. For each fixed $\eta>0$, the function

$$
h_{\eta}(\phi)= \begin{cases}\frac{p(\phi \eta)-p(\eta)}{\phi^{2}-1}, & \text { for } \phi \neq 1(\phi>0) \\ \frac{1}{2} \eta p^{\prime}(\eta), & \text { for } \phi=1\end{cases}
$$

is continuous and strictly monotone increasing for $\phi>0$.

Proof. The continuity of $h_{\eta}(\phi)$ follows by L'Hospital's rule (cf. Remark 6.3). To prove that $h_{\eta}(\phi)$ is strictly monotone increasing we compute its derivative in $\phi$,

$$
h_{\eta}^{\prime}(\phi)=\frac{\left(\phi^{2}-1\right) \eta p^{\prime}(\phi \eta)-2 \phi[p(\phi \eta)-p(\eta)]}{\left(\phi^{2}-1\right)^{2}} \quad \text { for } \quad \phi \neq 1
$$

Thus we only need to prove that for all $\phi \neq 1(\phi>0)$,

$$
g_{\eta}(\phi) \equiv\left(\phi^{2}-1\right) \eta p^{\prime}(\phi \eta)-2 \phi[p(\phi \eta)-p(\eta)]>0 .
$$

We compute

$$
\begin{aligned}
& g_{\eta}^{\prime}(\phi)=\left(\phi^{2}-1\right) \eta^{2} p^{\prime \prime}(\phi \eta)-2[p(\phi \eta)-p(\eta)] \\
& \lim _{\phi \rightarrow 1} \frac{g_{\eta}(\phi)}{(\phi-1)^{2}}=\eta\left[\eta p^{\prime \prime}(\eta)-p^{\prime}(\eta)\right] .
\end{aligned}
$$

From Lemma 3.3 and the fact that $\lim _{\eta \rightarrow 0} k(\eta)=1$ we see that $k(\eta)<1$ if $\eta>0$, i.e.,

$$
\frac{\eta p^{\prime \prime}(\eta)}{p^{\prime}(\eta)}<1 \quad \text { if } \quad \eta>0
$$


Hence (7.12) yields

$$
g_{\eta}(\phi)>\frac{1}{2}(\phi-1)^{2} \eta\left[\eta p^{\prime \prime}(\eta)-p^{\prime}(\eta)\right]>0
$$

if $\phi \in(1-\delta, 1+\delta) \backslash\{1\}$, for some small $\delta>0$.

Assume now that (7.10) is not true for all $\phi \geq 1+\delta$. Then there exists a $\phi_{0}>1+\delta$ such that $g_{\eta}(\phi)>0$ if $1<\phi<\phi_{0}$, and $g_{\eta}\left(\phi_{0}\right)=0$; this implies that $g_{\eta}^{\prime}\left(\phi_{0}\right) \leq 0$. On the other hand we have, by (7.11),

$$
\begin{array}{rlr}
g_{\eta}^{\prime}\left(\phi_{0}\right) & =\left(\phi_{0}^{2}-1\right) \eta^{2} p^{\prime \prime}\left(\phi_{0} \eta\right)-2\left[p\left(\phi_{0} \eta\right)-p(\eta)\right] \\
& =\left(\phi_{0}^{2}-1\right) \eta^{2} p^{\prime \prime}\left(\phi_{0} \eta\right)-\frac{\phi_{0}^{2}-1}{\phi_{0}} \eta p^{\prime}\left(\phi_{0} \eta\right) & \left(\text { since } g_{\eta}\left(\phi_{0}\right)=0\right) \\
& =\frac{\left(\phi_{0}^{2}-1\right) \eta}{\phi_{0}}\left[\phi_{0} \eta p^{\prime \prime}\left(\phi_{0} \eta\right)-p^{\prime}\left(\phi_{0} \eta\right)\right]>0 \quad & \text { by }(7.13),
\end{array}
$$

a contradiction.

Similarly one can prove that $(7.10)$ is true for all $0<\phi<1$.

THEOREM 7.2. The following properties hold:

$$
\begin{aligned}
& \frac{\partial}{\partial \bar{\beta}} \Lambda_{0}^{*}[\bar{\beta}, \gamma]<0, \\
& \frac{\partial}{\partial \gamma} \Lambda_{0}^{*}[\bar{\beta}, \gamma]>0 \\
& \lim _{\bar{\beta} \rightarrow \infty} \Lambda_{0}^{*}[\bar{\beta}, \gamma]=-\infty .
\end{aligned}
$$

Proof. Since

$$
\left.\frac{\partial f}{\partial \eta}\right|_{\eta=\eta_{0}}=0
$$

we have, by (7.7) and (7.4),

$$
\frac{\partial \Lambda_{0}^{*}}{\partial \bar{\beta}}=\frac{\partial f}{\partial \bar{\beta}}\left(\eta_{0}, \bar{\beta}, \phi\right)=\frac{1}{\lambda \bar{\sigma}} \frac{p\left(\phi \eta_{0}\right)-p\left(\eta_{0}\right)}{\phi^{2}-1}<0,
$$

where last inequality follows from the facts that $\bar{\sigma}>0$ and that the function $p(\eta)$ is strictly monotone decreasing. Hence (7.14) is proved. Similarly,

$$
\begin{aligned}
\frac{\partial \Lambda_{0}^{*}}{\partial \gamma} & =\frac{\partial \Lambda_{0}^{*}}{\partial \phi} \cdot \frac{\partial \phi}{\partial \gamma}=\frac{1}{2 \sqrt{\gamma \lambda}} \frac{\partial f}{\partial \phi}\left(\eta_{0}, \bar{\beta}, \phi\right) \\
& =\frac{1}{2 \sqrt{\gamma \lambda}} \frac{\bar{\beta}}{\lambda \bar{\sigma}} h_{\eta_{0}}^{\prime}(\phi)>0 \quad \text { (by Lemma 7.1) }
\end{aligned}
$$

and (7.15) follows. Finally, to prove (7.16) we take any $0<\bar{\eta}<\infty$ and write

$$
\Lambda_{0}^{*}=f\left(\eta_{0}, \bar{\beta}, \phi\right) \leq f(\bar{\eta}, \bar{\beta}, \phi)=p(\bar{\eta})+\frac{\bar{\beta}}{\lambda \bar{\sigma}} \cdot \frac{p(\phi \bar{\eta})-p(\bar{\eta})}{\phi^{2}-1} .
$$


Since $p(\eta)$ is strictly monotone decreasing and $\bar{\sigma}>0$, we see that

$$
\frac{1}{\lambda \bar{\sigma}} \frac{p(\phi \bar{\eta})-p(\bar{\eta})}{\phi^{2}-1}<0
$$

and (7.16) readily follows.

Theorem 7.3. Assume that (7.2) holds and that either $0<\Lambda_{0}<\frac{1}{3}$ or $\Lambda_{0}^{*}<\Lambda_{0}<0$. Then

$$
\begin{array}{rlrl}
\frac{\partial}{\partial \bar{\beta}} \eta[\bar{\beta}, \gamma]<0, & & \frac{\partial}{\partial \gamma} \eta[\bar{\beta}, \gamma]>0, \\
\frac{\partial}{\partial \bar{\beta}} \eta^{-}[\bar{\beta}, \gamma]<0, & \frac{\partial}{\partial \gamma} \eta^{-}[\bar{\beta}, \gamma]>0, \\
\frac{\partial}{\partial \bar{\beta}} \eta^{+}[\bar{\beta}, \gamma]>0, & \frac{\partial}{\partial \gamma} \eta^{+}[\bar{\beta}, \gamma]<0, \\
\lim _{\bar{\beta} \rightarrow \infty} \eta[\bar{\beta}, \gamma]=0, & \\
\lim _{\bar{\beta} \rightarrow \infty} \eta^{-}[\bar{\beta}, \gamma]=0, & \\
\lim _{\bar{\beta} \rightarrow \infty} \eta^{+}[\bar{\beta}, \gamma]=\infty, &
\end{array}
$$

Proof. By definition, $\eta=\eta(\bar{\beta}, \phi)$ is the solution of the equation

$$
f(\eta, \bar{\beta}, \phi)=\Lambda_{0} .
$$

Differentiating this equation with respect to $\bar{\beta}$ and $\phi$ respectively, we get

$$
\begin{aligned}
& \frac{\partial \eta(\bar{\beta}, \phi)}{\partial \bar{\beta}}=-\frac{\left.\frac{\partial f}{\partial \bar{\beta}}\right|_{\eta=\eta(\bar{\beta}, \phi)}}{\left.\frac{\partial f}{\partial \eta}\right|_{\eta=\eta(\bar{\beta}, \phi)}}=-\frac{\left.\frac{1}{\lambda \bar{\sigma}} h_{\eta}(\phi)\right|_{\eta=\eta(\bar{\beta}, \phi)}}{\left.\frac{\partial f}{\partial \eta}\right|_{\eta=\eta(\bar{\beta}, \phi)}} \\
& \frac{\partial \eta(\bar{\beta}, \phi)}{\partial \phi}=-\frac{\left.\frac{\bar{\beta}}{\lambda \bar{\sigma}} h_{\eta}^{\prime}(\phi)\right|_{\eta=\eta(\bar{\beta}, \phi)}}{\left.\frac{\partial f}{\partial \eta}\right|_{\eta=\eta(\bar{\beta}, \phi)}} .
\end{aligned}
$$

Since $\left.\frac{\partial f}{\partial \eta}\right|_{\eta=\eta(\bar{\beta}, \phi)}<0$ (by Theorem 3.5), the inequalities in (7.18) follow immediately from (7.8), (7.17) and from Lemma 7.1. The inequalities (7.19), (7.20) can be proved in a similar way. Next let us prove (7.21). From (7.18) we see that

$$
\eta^{*} \equiv \lim _{\bar{\beta} \rightarrow \infty} \eta[\bar{\beta}, \gamma]
$$

exists and is nonnegative and finite. If this number is positive then, by dividing (7.24) by $\bar{\beta}$ and letting $\bar{\beta} \rightarrow \infty$, we obtain

$$
\frac{1}{\lambda \bar{\sigma}} \frac{p\left(\phi \eta^{*}\right)-p\left(\eta^{*}\right)}{\phi^{2}-1}=0
$$


which is a contradiction since $p(\eta)$ is strictly monotone decreasing. Hence we must have $\eta^{*}=0$, and (7.21) is proved. The relations (7.22) and (7.23) can be proved in the same way.

Let us now examine the results obtained above from the point of view of medical treatment of tumors. If $0<\Lambda_{0}<\frac{1}{3}$ then there exists a unique dormant tumor, and it is globally asymptotically stable; Theorem 7.3 tells us that its radius decreases as $\bar{\beta}$ is increased. If $\Lambda_{0}<0$ then the tumor becomes unbounded (and $R(t) \rightarrow \infty$ as $t \rightarrow \infty$ ) when there is no inhibitor. However, in the presence of inhibitor, Theorem 7.2 tells us that if the external concentration $\bar{\beta}$ exceeds a certain critical number $\beta^{*}$ then two dormant states appear with radii $R_{s}^{-}$and $R_{s}^{+}, R_{s}^{-}<R_{s}^{+} ; R_{s}^{-}$is asymptotically stable, and $R(t) \rightarrow R_{s}^{-}$as $t \rightarrow \infty$, provided $R_{0}<R_{s}^{+}$. On the other hand if $R_{0}>R_{s}^{+}$then (at least for some initial data) $R(t)$ grows to $\infty$ as $t \rightarrow \infty$. Given initial radius $R_{0}$, the last conclusion of Theorem 7.3 asserts that we can increase $\bar{\beta}$ so that $R_{s}^{+}=R_{s}^{+}(\bar{\beta})$ (which is dependent on $\bar{\beta}$ ) will satisfy $R_{s}^{+}(\bar{\beta})>R_{0}$, and then indeed $R(t) \rightarrow R_{s}^{-}(\bar{\beta})$ as $t \rightarrow \infty$. The constant $\beta^{* *}$ for which $R_{s}^{+}(\bar{\beta})>R_{0}$ when $\bar{\beta}>\beta^{* *}$ will of course depend on $R_{0}$, but $\beta^{*}$ does not depend on $R_{0}$. Finally, when $\bar{\beta}$ keeps increasing, $R_{s}^{-}(\bar{\beta})$ keeps decreasing, and $R_{s}^{-}(\bar{\beta}) \rightarrow 0$ as $\bar{\beta} \rightarrow \infty$.

In conclusion, by increasing the amount of drug concentration $\bar{\beta}$ we can always decrease the tumor and in fact, render its limiting size arbitrarily small; the smallest concentration that ensures containment of the tumor is $\bar{\beta}=\beta^{* *}$, and it is a function of its initial radius $R_{0}$.

Theorems 7.2 and 7.3 also show that decreasing $\gamma$ has a similar effect as increasing $\bar{\beta}$ : the smaller the $\gamma$, the more effective the drug is; if $\gamma$ is decreased then the limiting $R(t)$ is also decreased.

REmark 7.1. So far we have assumed that (7.2) holds. Consider next the case

$$
\bar{\sigma}<0 \quad \text { (Figures } 3 \text { and } 4 \text { ). }
$$

If $0<\Lambda_{0}<\frac{1}{3}$ then there is just one dormant state with normalized radius $\eta$, and if $\frac{1}{3}<\Lambda_{0}<\Lambda_{0}^{* *}$ then there are two dormant states with normalized radii $\eta^{-}$and $\eta^{+}$, where $\eta^{-}<\eta^{+}$. Proceeding as in the case of $(7.2)$, one can prove that

$$
\begin{array}{lll}
\frac{\partial}{\partial \bar{\beta}} \Lambda_{0}^{* *}[\bar{\beta}, \gamma]>0, & \frac{\partial}{\partial \gamma} \Lambda_{0}^{* *}[\bar{\beta}, \gamma]<0, \\
\lim _{\bar{\beta} \rightarrow \infty} \Lambda_{0}^{* *}[\bar{\beta}, \gamma]=\infty, & \\
\frac{\partial}{\partial \bar{\beta}} \eta[\bar{\beta}, \gamma]>0, & \frac{\partial}{\partial \gamma} \eta[\bar{\beta}, \gamma]<0, \\
\frac{\partial}{\partial \bar{\beta}} \eta^{-}[\bar{\beta}, \gamma]<0, & \frac{\partial}{\partial \gamma} \eta^{-}[\bar{\beta}, \gamma]>0, \\
\frac{\partial}{\partial \bar{\beta}} \eta^{+}[\bar{\beta}, \gamma]>0, & \frac{\partial}{\partial \gamma} \eta^{+}[\bar{\beta}, \gamma]<0, \\
\lim _{\bar{\beta} \rightarrow \infty} \eta[\bar{\beta}, \gamma]=\infty, & \\
\lim _{\bar{\beta} \rightarrow \infty} \eta^{-}[\bar{\beta}, \gamma]=0, & \\
\lim _{\bar{\beta} \rightarrow \infty} \eta^{+}[\bar{\beta}, \gamma]=\infty . &
\end{array}
$$

In the present case of $\bar{\sigma}<0, \eta^{-}$is stable whereas $\eta$ and $\eta^{+}$are unstable. Increasing $\bar{\beta}$ has a 
positive effect: if $\bar{\beta}$ is increased beyond a critical number $\beta^{* *}$ so that

$$
\frac{\eta}{\sqrt{\lambda}}>R_{0}, \quad \text { or } \quad \frac{\eta^{+}}{\sqrt{\lambda}}>R_{0},
$$

then the tumor will be contained and $R(t)$ will converge to either 0 (if $0<\Lambda_{0}<\frac{1}{3}$ ) or $\eta^{-} / \sqrt{\lambda}$ (if $\frac{1}{3}<\Lambda_{0}<\Lambda_{0}^{* *}$ ). Here again a drug with smaller $\gamma$ is more effective: if $\gamma$ is decreased then the limiting $R(t)$ is also decreased.

We finally note that the case $\bar{\sigma}=0$ can be handled as a limit case of either $\bar{\sigma}>0$ or $\bar{\sigma}<0$.

8. Conclusion. We have shown that, for fixed $\Lambda_{0}$, the number of dormant tumors depend on the parameters $\Lambda_{1}, \phi$ defined in (1.19), or on the intrinsic inhibitor-parameter $\gamma$ and its external concentration $\bar{\beta}$. There may be one, two or no dormant states. In the cases where dormant states exist, we determined by rigorous mathematical analysis which of them is asymptotically stable and which is unstable; when two dormant states exist, the smaller one is stable and the larger one is unstable. We established monotonic dependence of the tumor's radius on the parameters $\bar{\beta}$ and $\gamma$. Our analysis suggests how the external concentration $\bar{\beta}$ should be chosen:

$\bar{\beta}$ should exceed the level $\beta^{* *}$ for which the initial tumor radius $R_{0}$ becomes smaller than the radius of the unstable dormant tumor. This will ensure that $R(t)$ will converge either to the radius of the stable dormant tumor (Figures 1, 2, and $4(\mathrm{~b})$ ) or to zero (Figures 3 and $4(\mathrm{a})$ ). By further increasing $\bar{\beta}$ we can decrease the size of the stable dormant tumor, which is of course always desirable if one may neglect side-effects. We have also established that the drug is more effective the smaller its parameter $\gamma$ is, provided again that one may neglect side-effects.

The present paper does not consider the side-effects of increasing the doze or strength of the inhibitor. At present, the decision for treatment of solid tumors rests on experience based on obtaining the optimal ratio of cure rates to undesirable responses.

Acknowledgement. The first author is supported by China Scholarship Council and the Institute for Mathematics and its Applications. The second author is partially supported by the National Science Foundation Grant DMS \# 94-01251.

\section{REFERENCES}

[1] N.M. Byrne and M.A.J. Chaplain, Growth of nonnecrotic tumors in th presence and absence of inhibitors, Math. Biosci., 130 (1995), 151-181.

[2] H.P. Greenspan, Models for the growth of a solid tumor by diffusion, Studies in Appl. Math., 52 (1972), 317-340.

[3] J.A. Adam, A simplified mathematical model of tumor growth, Math. Biosci., 81 (1986), 229-244.

[4] J.A. Adam, A mathematical model of tumor growth. II. Effects of geometry and spatial nonuniformity on stability, Math. Biosci., 86 (1987), 183-211.

[5] N.M. Byrne and M.A.J. Chaplain, Growth of necrotic tumors in the presence and absence of inhibitors, Math. Biosci., 135 (1996), 187-216.

[6] N.M. Byrne, The effect of time delays on the dynamics of a vascular tumor growth, Math. Biosci., 144 (1997), 83-117.

[7] N.F. Britton and M.A.J. Chaplain, A qualitative analysis of some models of tissue growth, Math. Biosci., 113 (1993), 77-89. 
[8] J.A. Adam and N. Bellomo, A survey of models for tumor-immune system dynamics, Birkhäuser, Boston, MA, 1997.

[9] A. Friedman and F. Reitich, Analysis of a mathematical model for the growth of tumors, J. Math. Biol., 38 (1999), 262-284.

[10] A. Friedman, Partial differential equations of parabolic type, Prentice-Hall, Englewood Cliffs, N.J., 1964. 